\title{
ANALYSIS OF A MULTIPLE-POROSITY \\ MODEL FOR SINGLE-PHASE FLOW \\ THROUGH NATURALLY FRACTURED \\ POROUS MEDIA
}

\author{
ANNA MARIA SPAGNUOLO AND STEVE WRIGHT
}

Received 28 May 2002 and in revised form 24 February 2003

A derivation of a multiple-porosity model for the flow of a single phase, slightly compressible fluid in a multiscale, naturally fractured reservoir is presented by means of recursive use of homagnetization theory. We obtain a model which generalizes the double-porosity model of Arbogast et al. (1990) to a flow system with an arbitrary finite number of scales.

\section{Introduction}

A model for single-phase flow in porous media that are hierarchically fissured in regular patterns was derived by a recursive asymptotic expansion technique in [16] and part of [24]. This work rigorously justifies that model. Through recursive homogenization, we extend the doubleporosity model in [5], which has one fracture system and a matrix (rock) block system, to a triple-porosity model that has two levels of fracture systems and a matrix block system. See $[9,22]$ for an introduction to homogenization theory. Then, a multiple-porosity model with $N$ levels of fracture systems and a matrix block system is derived, resulting in a general $(N+1)$-scale model.

A dual-porosity concept was first introduced in $[8,25]$ using a specific transmissibility function (see [7]) for the interaction of the matrixfracture flow. For petroleum-reservoir engineering problems, a new treatment of the coupling of the flow through the fracture system with that in the matrix system was introduced over the past two decades in $[2,3,4,5,6,13,14,17,18,19]$. The models discussed in this work are based on these ideas. Our focus on the nested levels of fracture systems is appropriate for further studies on high-level nuclear waste transport

Copyright @ 2003 Hindawi Publishing Corporation Journal of Applied Mathematics 2003:7 (2003) 327-364

2000 Mathematics Subject Classification: 76S05

URL: http:/ /dx.doi.org/10.1155/S1110757X03205143 


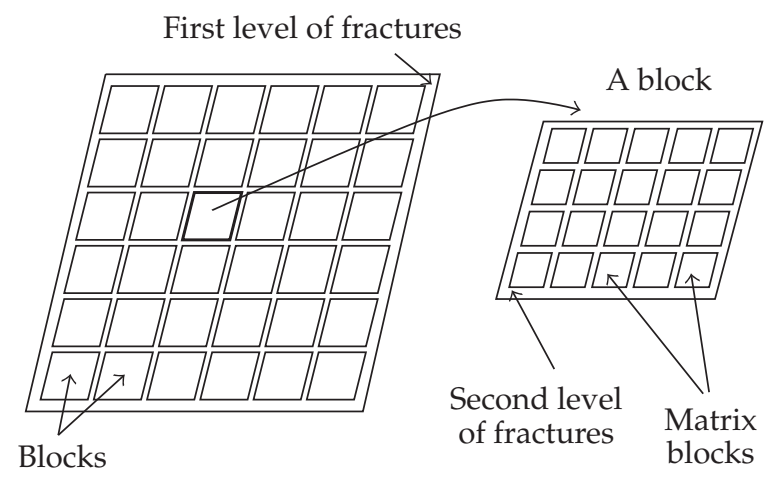

FIGURE 1.1. The periodic structure in a reservoir exhibiting two levels of fractures.

in porous media. It is the long-time scales, due to the length of the halflives of some high-level nuclear elements, that allow for the possibility of nested levels of fracture systems in porous media (see $[15,20]$ ).

Since we are interested in the mathematical details of the problem here, we refer to the introductions of $[16,24]$ for further details on the applicability of the models.

This work deals with modeling a single-phase, constant-compressibility fluid flowing in a geometrically complicated structure given (initially) by a naturally fractured reservoir that has a hierarchy of fracture systems, with the first being defined by an interconnected system of planar fractures dividing the reservoir into a collection of disjoint blocks. A second system of fractures divides each of the previous blocks into a collection of equally sized smaller blocks, and so forth, until a last level is reached in which the disjoint blocks behave as a collection of disjoint unfractured matrix blocks. The geometric structure is idealized by the assumption that each fracture system is periodic. See Figure 1.1 for a cross-sectional view of the idealized reservoir in the case of two levels of fracturing.

We begin by posing the flow equations on three different scales of the domain. This involves using three different porosity and permeability coefficients, one for each scale, since the fluid flows more readily through the fracture systems than it does through the matrix blocks. Via a parameter $\varepsilon_{1}$, which represents the linear size of a matrix block and half of its surrounding fractures, we first homogenize the flow equations on the smallest level of fractures and the matrix blocks. This gives an overall fracture flow in each of the fractured blocks. The porous matrix blocks provide a source term to the surrounding system of smallscale fractures which, after homogenization, are treated as a continuous 
porous medium. Thus, a continuous medium approach takes place between the smallest level of fractures and the matrix blocks. This is a scaled mesoscopic description since the equations depend on the parameter $\varepsilon_{0}$, which represents the linear size of the scaled fractured blocks. Following this averaging, we couple the equations for flow in the largest level of fractures with the fractured blocks, each of which is represented now by a double-porosity system. Averaging the flow in the first level of fractures then gives a macroscopic description of the flow in a threesheeted covering of the domain; this extends the concepts of the models of [13]. Thus, the first level of fractures is now smoothed out to cover the entire domain and the blocks interact with the first level of fractures as sources, while the behavior of the flow on a first-level block is that of a double-porosity system. Overall, the system can be characterized as a triple-porosity model.

The $(N+1)$-scale analysis discussed in this paper can be used as a tool for analyzing problems with multiple scales of periodicity (i.e., homogeneous, hierarchically organized media). But in the presence of heterogeneities, [11] addresses two-scale convergence in the mean and includes applications to randomly fractured media [21]. However, such an approach does not apply immediately to heterogeneities with multiple scales of correlation, which is the case for many natural porous media. The extension of the $(N+1)$-scale approach introduced here is hence an alternative that may improve our understanding of the flow phenomena in some natural porous media.

We first present the triple-porosity model in order to illustrate, in a simpler and more readily understandable situation, the general techniques that are necessary for the $(N+1)$-scale model. However, intermediate source terms that are not present in the triple-porosity model appear in the $(N+1)$-scale model and they require additional arguments.

The organization of the paper is as follows. In Section 2, the assumptions, notation, and description of the triple-porosity reservoir are given. Also, two dilation and two location operators are defined. In Section 3, the microscale model, which involves both the intermediate and microscopic levels, is formulated using the parameters $\varepsilon_{1}$ and $\varepsilon_{0}$ described above. The coefficients are precisely defined on the appropriate parts of $\Omega$. Next, in Section 4, the weak formulation of the microscale model is given, well-posedness is proven, a priori estimates are derived, and several technical lemmas regarding the dilation operators are presented. Then, the convergence results for the first homogenization $\left(\varepsilon_{1} \rightarrow 0\right)$ lead to a well-posed mesoscopic system of equations in Section 5 . Then, in Section 6, a completely new well-posed problem is formulated in terms of the parameter $\varepsilon_{0}$, using the resulting model in Section 5 with a new boundary condition that conserves mass flux. In Section 7, a general 
$(N+1)$-scale problem with $N$ levels of fractures and the matrix (porous) level is presented. Previous lemmas and theorems are generalized in this section, with the double- and triple-porosity models serving as base cases for the homogenization procedure. The final well-posed system of equations is presented for the finite scale. Finally, in Section 8, concluding remarks on generalizations of the model are made.

\section{Notation, assumptions, and preliminary lemmas}

We begin this section by defining the nested periodic structure of the domain $\Omega$ in the presence of $N$ levels of fractures. First, for $i=0, \ldots$, $N-1$, let $Y_{i}$ be a parallelepiped and let $\delta_{i} \in(0,1)$ be such that $\left|Y_{0}\right| \ll$ $\delta_{0}|\Omega|$ and, for $i=1, \ldots, N-1,\left|Y_{i}\right| \ll \delta_{i}\left|Y_{i-1}\right|$. Then, with $Y_{-1 B}=\Omega$, define $A^{\text {if }}$ to be a finite lattice containing the origin such that

$$
\bar{Y}_{(i-1) B}=\bigcup_{c^{i} \in A^{i f}}\left(\bar{Y}_{i}+c^{i}\right),
$$

where

$$
Y_{i}=Y_{i B} \cup \partial Y_{i B} \cup Y_{i F}
$$

Now, extend the lattice $A_{i f}$ into an infinite lattice $A^{i}$, containing the origin and define $\Omega_{F}^{\varepsilon_{0}}, \Omega_{B}^{\varepsilon_{0}}$ by

$$
\Omega_{F}^{\varepsilon_{0}}=\Omega \cap\left(\bigcup_{c^{0} \in A^{0}} \varepsilon_{0}\left(Y_{0 F}+c^{0}\right)\right), \quad \Omega_{B}^{\varepsilon_{0}}=\Omega \cap\left(\bigcup_{c^{0} \in A^{0}} \varepsilon_{0}\left(Y_{0 B}+c^{0}\right)\right),
$$

and for $i=1,2, \ldots, N-1$, define

$$
\begin{aligned}
\Omega_{B, H}^{\varepsilon_{0} \cdots \varepsilon_{i}}=\Omega_{B, B}^{\varepsilon_{0} \cdots \varepsilon_{i-1}} \cap\left(\bigcup_{c^{0} \in A^{0}} \cdots \bigcup_{c^{i} \in A^{i}}\right. & \left(\varepsilon_{0} \cdots \varepsilon_{i}\left(Y_{i H}+c^{i}\right)\right. \\
& \left.\left.+\varepsilon_{0} \cdots \varepsilon_{i-1} c^{i-1}+\cdots+\varepsilon_{0} c^{0}\right)\right),
\end{aligned}
$$

where $H=B$ or $F$ (see Figure 2.1).

Since we are assuming that there are $N$ levels of fractures in $\Omega$, we let $\varepsilon_{0}, \ldots, \varepsilon_{N-1}$ be the parameters associated with the homogenization. In order to define dilation operators that incorporate each of these parameters, we proceed as follows. For $i=0, \ldots, N-1$, let

$$
c^{i, \varepsilon_{i}}: Y_{(i-1) B} \longrightarrow \varepsilon_{i} A^{i},
$$




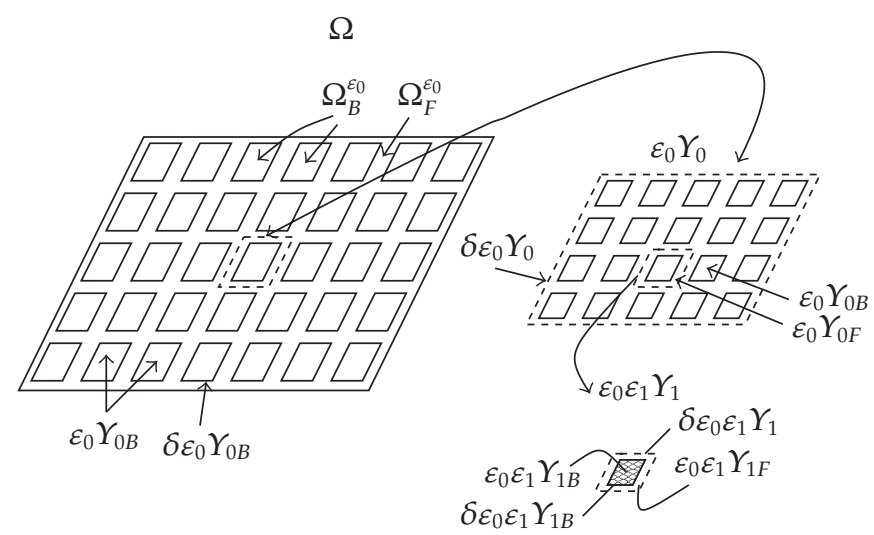

FIGURE 2.1. The parts of the reservoir.

where, for $x_{i} \in Y_{(i-1) B}, c^{i, \varepsilon_{i}} \in \varepsilon_{i} A^{i}$ is the lattice translation point of the $\varepsilon_{i} Y_{i^{-}}$ cell containing $x_{i}$, that is, $c^{i, \varepsilon_{i}}$ is the lattice translation vector such that $x_{i} \in \varepsilon_{i} Y_{i}+c^{i, \varepsilon_{i}}\left(x_{i}\right)$.

Then define the dilation operator ${ }^{(i)}$, from the set of functions defined on $\Omega_{B, H}^{\varepsilon_{0} \cdots \varepsilon_{i-1}}(H=B, F)$ to functions defined on $\Omega \times \cdots \times \Upsilon_{(i-2) B} \times \Upsilon_{(i-1) H}$, by

$$
\begin{gathered}
f^{\sim(i)}\left(x_{0}, \ldots, x_{i}\right)=f\left(\left(\varepsilon_{0} \cdots \varepsilon_{i-1}\right) x_{i}+\left(\varepsilon_{0} \cdots \varepsilon_{i-2}\right) c^{(i-1), \varepsilon_{i-1}}\left(x_{i-1}\right)\right. \\
\left.+\cdots+\varepsilon_{0} c^{1, \varepsilon_{1}}\left(x_{1}\right)+c^{0, \varepsilon_{0}}\left(x_{0}\right)\right) .
\end{gathered}
$$

We also make heavy use of the definition of ${ }^{\sim(i)}$ for functions defined on $\Omega_{B, B}^{\varepsilon_{0} \cdots \varepsilon_{i-2}}$ in the same way, except that in this case, ${ }^{\sim(i)}$ maps to functions defined on $\Omega \times Y_{0 B} \times \cdots \times Y_{(i-2) B} \times Y_{i-1}$.

For convenience, we recursively define the following location operators:

$$
L_{-1}\left(x_{0}\right)=x_{0}, \quad L_{0}\left(x_{0}\right)=\frac{x_{0}-c^{0, \varepsilon_{0}}\left(x_{0}\right)}{\varepsilon_{0}} \in \bar{Y}_{0} \quad \text { for } x_{0} \in \Omega,
$$

and, in general, for $x_{0} \in \Omega_{B, B}^{\varepsilon_{0} \cdots \varepsilon_{i-1}}$,

$$
L_{i}\left(x_{0}\right)=\frac{L_{i-1}\left(x_{0}\right)-c^{i, \varepsilon_{i}}\left(L_{i-1}\left(x_{0}\right)\right)}{\varepsilon_{i}} \in \bar{Y}_{i} \quad \text { for } i=1, \ldots, N-1 .
$$

Let $\rho_{i}^{\varepsilon_{0} \cdots \varepsilon_{i}}$, for $i=0, \ldots, N-1$, be the density of the fluid on the $(i+1)$ st level of fractures, and let $\rho_{N}^{\varepsilon_{0} \cdots \varepsilon_{N-1}}$ be the density of the fluid in the porous matrix blocks. The following definitions reflect the nested periodic property of $\Omega$. Let $\phi_{0}$ and $K_{0}$ be the scalar porosity and scalar permeability, respectively, on the first level of fractures, extended throughout $\Omega$. For 
$i=1, \ldots, N-1$, we define the porosities $\phi_{i}$ and permeabilities $K_{i}$ on the $(i+1)$ st level of fractures as follows. First, we assume that $\phi_{i}$ and $K_{i}$ are defined on $Y_{(i-1) B}$. So, $\phi_{i}=\phi_{i}\left(x_{i}\right)$ and $K_{i}=K_{i}\left(x_{i}\right)$, for $x_{i} \in Y_{(i-1) B}$. Then, we extend these definitions to all of $\Omega_{B, B}^{\varepsilon_{0} \cdots \varepsilon_{i-1}}$ by defining $\phi_{i}^{\varepsilon_{0} \cdots \varepsilon_{i-1}}\left(x_{0}\right)=$ $\phi_{i}\left(L_{i-1}\left(x_{0}\right)\right)$ and $K_{i}^{\varepsilon_{0} \cdots \varepsilon_{i-1}}\left(x_{0}\right)=K_{i}\left(L_{i-1}\left(x_{0}\right)\right)$. Similarly, we first define $\phi_{N}$ and $\mathbf{K}_{N}$ on $Y_{(N-1) B}$, and then extend their definitions to $\Omega_{B, B}^{\varepsilon_{0} \cdots \varepsilon_{N-1}}$ by defining $\phi_{N}^{\varepsilon_{0} \cdots \varepsilon_{N-1}}\left(x_{0}\right)=\phi_{N}\left(L_{N-1}\left(x_{0}\right)\right)$ and $\mathbf{K}_{N}^{\varepsilon_{0} \cdots \varepsilon_{N-1}}\left(x_{0}\right)=\mathbf{K}_{N}\left(L_{N-1}\left(x_{0}\right)\right)$. All coefficients are uniformly positive and bounded, and $\mathbf{K}_{N}$ is a bounded, symmetric, positive-definite tensor.

In order to carry out our recursive homogenization process, we require that the fracture and matrix geometry satisfy

$$
\partial \Omega \subseteq \partial \Omega_{F}^{\varepsilon_{0}}, \quad \partial \Omega_{B, F}^{\varepsilon_{0} \cdots \varepsilon_{i-1}} \subseteq \partial \Omega_{B, F}^{\varepsilon_{0} \cdots \varepsilon_{i}}, \quad i=1, \ldots, N-1 .
$$

Let $J=(0, T)$ be the time interval of interest. Also, throughout this paper, we denote by $n_{D}$ the outward unit normal to the boundary of $D$, where $D$ is the relevant domain.

We begin the study of our model at the microscopic level, which consists of equations describing Darcy flow on all parts of $\Omega$, that is, we will pose the flow equations separately on the disjoint regions that compose the domain. For the homogenization process, the equations on the different parts of $\Omega$ will have to be scaled appropriately to conserve flow, just as was necessary in the derivation of the double-porosity model (see [13]). Actually, it is convenient to scale the equations on $\Omega_{B, B}^{\varepsilon_{0} \varepsilon_{1}}$ with respect to the equations on $\Omega_{B, F}^{\varepsilon_{0} \varepsilon_{1}}$ and then again with respect to those on $\Omega_{F}^{\varepsilon_{0}}$. This will allow us to derive, recursively, a triple-porosity model through rigorous homogenization.

For convenience, assume that gravity is negligible. This assumption is only used to simplify the presentation. A note regarding the inclusion of gravity is made just after the derivation of the equations in terms of the density of the fluid. Assume that the fluid has viscosity $\mu$ and constant (small) compressibility $c$, so that the equation of state is given by

$$
d \rho=c \rho d p,
$$

where $\rho$ is the density of the fluid and $p$ is its pressure. In a singleporosity model, if $\mathbf{K}$ is the permeability (which can be a tensor), then the volumetric flow rate $v$ of the fluid is given by Darcy's law:

$$
v=-\frac{\mathbf{K}}{\mu} \nabla p .
$$


If $\phi$ denotes the porosity of the medium, the conservation of mass requires that

$$
\phi \frac{\partial \rho}{\partial t}+\nabla \cdot(\rho v)=S
$$

where $S$ is the external source. Rewriting this in terms of $\rho$, we obtain

$$
\phi \frac{\partial \rho}{\partial t}-\nabla \cdot\left(\frac{\mathbf{K}}{\mu c} \nabla \rho\right)=S .
$$

We remark that if the gravity term $-\nabla \cdot\left((\mathbf{K} / \mu c)\left(c g \rho^{2}\right)\right)$ is added to the left-hand side of the above equation, then everything that follows holds if we linearize the equation as in [5] by defining a reference density $\rho_{\text {ref }}$ and approximating the effects of gravity by $\rho^{2} \approx \rho_{\text {ref }}\left(2 \rho-\rho_{\text {ref }}\right)$.

The verification of our homogenization procedure will make a crucial use of the following technical lemmas. In the interest of brevity, we omit their proofs.

Lemma 2.1. For $\psi, \varphi \in L^{2}\left(\Omega_{B, r}^{\varepsilon_{0} \cdots \varepsilon_{i-1}}\right)$, where $r=B, F$, or blank, and $\Omega_{B,}^{\varepsilon_{0} \cdots \varepsilon_{i-1}} \equiv$ $\Omega_{B, B}^{\varepsilon_{0} \cdots \varepsilon_{i-2}}$,

$$
\begin{aligned}
\left(\psi^{\sim(i)}, \varphi^{\sim(i)}\right)_{\Omega \times Y_{0 B} \times \cdots \times Y_{(i-2) B} \times Y_{(i-1) r}} & =\left|Y_{0}\right|\left|Y_{1}\right| \cdots\left|Y_{i-1}\right|(\psi, \varphi)_{\Omega_{B, r}^{\varepsilon_{0} \cdots \varepsilon_{i-1}},} \\
\nabla_{x_{i}} \psi^{\sim(i)} & =\varepsilon_{0} \cdots \varepsilon_{i-1}(\nabla \psi)^{\sim(i)}, \\
\left\|\psi^{\sim(i)}\right\|_{L^{2}\left(\Omega \times Y_{0 B} \times \cdots \times Y_{(i-1) r}\right)} & =\left(\left|Y_{0}\right|\left|Y_{1}\right| \cdots\left|Y_{i-1}\right|\right)^{1 / 2}\|\psi\|_{L^{2}\left(\Omega_{B, r}^{\varepsilon_{0} \cdots \varepsilon_{i-1}}\right)}, \\
\left\|\nabla_{x_{i}} \psi^{\sim(i)}\right\|_{L^{2}\left(\Omega \times Y_{0 B} \times \cdots \times Y_{(i-2) B} \times Y_{(i-1) r}\right)}= & \varepsilon_{0} \cdots \varepsilon_{i-1}\left(\left|Y_{0}\right|\left|Y_{1}\right| \cdots\left|Y_{i-1}\right|\right)^{1 / 2} \\
& \times\|\nabla \psi\|_{L^{2}\left(\Omega_{B, r}^{\varepsilon_{B} \cdots \varepsilon_{i-1}}\right)}
\end{aligned}
$$

for $i=1, \ldots, N$.

Lemma 2.2. If $\psi \in L^{2}(\Omega)$, then the following holds strongly in $L^{2}\left(\Omega \times Y_{0 B} \times\right.$ $\left.\cdots \times Y_{(i-2) B} \times Y_{(i-1) r}\right)$ :

$$
\lim _{\varepsilon_{i-1} \rightarrow 0} \psi^{\sim(i)}=\psi^{\sim(i-1)},
$$

for $i=1, \ldots, N$, where $^{\sim(0)}$ is the identity operator.

In what follows, $\mathbf{e}_{j}$ denotes the $j$ th standard basis vector in the appropriate Euclidean space. 
334 Analysis of a multiple-porosity model

LEMMA 2.3. Let $f \in L^{2}(\Omega)$ and $g \in L^{2}\left(\Omega \times Y_{0 B} \times Y_{1 B} \times \cdots \times Y_{(i-2) B} \times Y_{i-1}\right)$.

Then, for $i=2,3, \ldots$,

$$
\begin{aligned}
& \int_{\Omega \times Y_{0 B} \times \cdots \times Y_{(i-2) B} \times Y_{i-1}} \tilde{f}^{(i)}\left(x_{0}, x_{1}, \ldots, x_{i}\right) g\left(x_{0}, x_{1}, \ldots, x_{i}\right) d x_{i} \cdots d x_{0} \\
& =\int_{\Omega_{B, B}^{\varepsilon_{0} \cdots \varepsilon_{i-2} \times Y_{0} \times Y_{1} \times \cdots \times Y_{i-1}}} f\left(x_{0}\right) g\left(\sum_{k=0}^{i-1}\left(\varepsilon_{k} x_{k+1}+c^{k, \varepsilon_{k}}\left(L_{k-1}\left(x_{0}\right)\right)\right) \mathbf{e}_{k}\right. \\
& \left.+L_{i-1}\left(x_{0}\right) \mathbf{e}_{i}\right) d x_{i} \cdots d x_{0}
\end{aligned}
$$

LEMMA 2.4. Let $f \in L^{2}(\Omega)$ and $g \in L^{2}\left(\Omega \times Y_{0 B} \times \cdots \times Y_{(i-1) B}\right)$. Then, for $i=$ $1,2, \ldots$,

$$
\begin{gathered}
\int_{\Omega \times Y_{0 B} \times \cdots \times Y_{(i-1) B}} \tilde{f}^{(i)}\left(x_{0}, x_{1}, \ldots, x_{i}\right) g\left(x_{0}, x_{1}, \ldots, x_{i}\right) d x_{i} \cdots d x_{0} \\
=\int_{\Omega_{B, B}^{\varepsilon_{0} \cdots \varepsilon_{i-1} \times Y_{0} \times \cdots \times Y_{i-1}}} f\left(x_{0}\right) g\left(\sum_{k=0}^{i-1}\left(\varepsilon_{k} x_{k+1}+c^{k, \varepsilon_{k}}\left(L_{k-1}\left(x_{0}\right)\right)\right) \mathbf{e}_{k}\right. \\
\left.+L_{i-1}\left(x_{0}\right) \mathbf{e}_{i}\right) d x_{i} \cdots d x_{0} .
\end{gathered}
$$

LEMMA 2.5. Let $f, g \in L^{2}\left(Y_{(i-2) B}\right)$. Then, with $Y_{-1 B} \equiv \Omega$, for $i=1,2, \ldots$, the following equation holds:

$$
\begin{aligned}
& \int_{Y_{(i-2) B} \times Y_{i-1}} f\left(\varepsilon_{i-1} y_{i}+c^{(i-1), \varepsilon_{i-1}}\left(x_{i-1}\right)\right) g\left(x_{i-1}\right) d y_{i} d x_{i-1} \\
& =\int_{Y_{(i-2) B} \times Y_{i-1}} f\left(x_{i-1}\right) g\left(\varepsilon_{i-1} y_{i}+c^{(i-1), \varepsilon_{i-1}}\left(x_{i-1}\right)\right) d y_{i} d x_{i-1} .
\end{aligned}
$$

\section{The initial microscopic equations for a triple-porosity model}

Denote by $\rho^{\varepsilon_{0}}\left(x_{0}, t\right)$ the density on $\Omega_{F}^{\varepsilon_{0}}$. Equations involving $\rho^{\varepsilon_{0}}$ will be posed once the initial homogenization has been completed (i.e., after letting $\left.\varepsilon_{1} \rightarrow 0\right)$. Let $\sigma^{\varepsilon_{0} \varepsilon_{1}}\left(x_{0}, t\right)$ and $\theta^{\varepsilon_{0} \varepsilon_{1}}\left(x_{0}, t\right)$ denote the densities on $\Omega_{B, F}^{\varepsilon_{0} \varepsilon_{1}}$ and $\Omega_{B, B}^{\varepsilon_{0} \varepsilon_{1}}$, respectively.

The assumptions made above lead to the sets (3.1) and (3.2) of equations for the micromodel. The scaling rules are explained immediately after the equations. 
On the second level of fractures,

$$
\begin{gathered}
\phi_{\sigma}^{\varepsilon_{0}} \frac{\partial \sigma^{\varepsilon_{0} \varepsilon_{1}}}{\partial t}-\varepsilon_{0}^{2} \nabla \cdot\left(\frac{K_{\sigma}^{\varepsilon_{0}}}{\mu c} \nabla \sigma^{\varepsilon_{0} \varepsilon_{1}}\right)=0 \quad \text { in } \Omega_{B, F}^{\varepsilon_{0} \varepsilon_{1}} \times J, \\
\frac{K_{\sigma}^{\varepsilon_{0}}}{\mu c} \nabla \sigma^{\varepsilon_{0} \varepsilon_{1}} \cdot n_{\Omega_{B, B}^{\varepsilon_{0} \varepsilon_{1}}}=\varepsilon_{1}^{2} \frac{K_{\theta}^{\varepsilon_{0} \varepsilon_{1}}}{\mu c} \nabla \theta^{\varepsilon_{0} \varepsilon_{1}} \cdot n_{\Omega_{B, B}^{\varepsilon_{0} \varepsilon_{1}}} \quad \text { on } \partial \Omega_{B, B}^{\varepsilon_{0} \varepsilon_{1}} \times J, \\
\frac{K_{\sigma}^{\varepsilon_{0}}}{\mu c} \nabla \sigma^{\varepsilon_{0} \varepsilon_{1}} \cdot n_{\Omega_{B}^{\varepsilon_{0}}}=0 \quad \text { on } \partial \Omega_{B}^{\varepsilon_{0}} \times J, \quad \sigma^{\varepsilon_{0} \varepsilon_{1}}=\rho_{\text {init }} \quad \text { in } \Omega_{B, F}^{\varepsilon_{0} \varepsilon_{1}} \times\{0\} .
\end{gathered}
$$

On the second level of matrix blocks,

$$
\begin{array}{cc}
\phi_{\theta}^{\varepsilon_{0} \varepsilon_{1}} \frac{\partial \theta^{\varepsilon_{0} \varepsilon_{1}}}{\partial t}-\varepsilon_{0}^{2} \varepsilon_{1}^{2} \nabla \cdot\left(\frac{\mathbf{K}_{\theta}^{\varepsilon_{0} \varepsilon_{1}}}{\mu c} \nabla \theta^{\varepsilon_{0} \varepsilon_{1}}\right)=0 & \text { in } \Omega_{B, B}^{\varepsilon_{0} \varepsilon_{1}} \times J, \\
\theta^{\varepsilon_{0} \varepsilon_{1}}=\sigma^{\varepsilon_{0} \varepsilon_{1}} \quad \text { on } \partial \Omega_{B, B}^{\varepsilon_{0} \varepsilon_{1}} \times J, \quad \theta^{\varepsilon_{0} \varepsilon_{1}}=\rho_{\text {init }} & \text { in } \Omega_{B, B}^{\varepsilon_{0} \varepsilon_{1}} \times\{0\} .
\end{array}
$$

The aim of this work is to use homogenization theory to rigorously determine the equations that describe the flow. Since we let $\varepsilon_{1} \rightarrow 0$ first, we do not, at this stage, consider the fluid flow across $\partial \Omega_{B}^{\varepsilon_{0}}$. Instead, we assume no-flow boundary conditions because we are interested in determining the interior behavior of the flow on $\Omega_{B}^{\varepsilon_{0}}$. Then, once the equations are discovered, we will impose boundary conditions on $\Omega_{B}^{\varepsilon_{0}}$ and develop a completely new system of partial differential equations that describe the flow. Then, we let $\varepsilon_{0} \rightarrow 0$ to obtain the final model. The case in which a flow across $\partial \Omega_{B}^{\varepsilon_{0}}$ is considered at the microscopic level will be taken up elsewhere.

\section{Preliminary analysis of the microscopic model}

Multiply (3.1) by a test function $\varphi \in H^{1}\left(\Omega_{B}^{\varepsilon_{0}}\right)$, and multiply (3.2) by a test function $\psi \in H_{0}^{1}\left(\Omega_{B, B}^{\varepsilon_{0} \varepsilon_{1}}\right)$. Then, use the boundary conditions and the divergence theorem to find that the weak form of the microscale model for fixed $\varepsilon_{1}$ is given by

$$
\begin{aligned}
\left(\phi_{\sigma}^{\varepsilon_{0}} \sigma_{t}^{\varepsilon_{0} \varepsilon_{1}}, \varphi\right)_{\Omega_{B, F}^{\varepsilon_{0} \varepsilon_{1}}}+ & \varepsilon_{0}^{2}\left(\frac{K_{\sigma}^{\varepsilon_{0}}}{\mu c} \nabla \sigma^{\varepsilon_{0} \varepsilon_{1}}, \nabla \varphi\right)_{\Omega_{B, F}^{\varepsilon_{0} \varepsilon_{1}}}+\varepsilon_{0}^{2} \varepsilon_{1}^{2}\left(\frac{\mathbf{K}_{\theta}^{\varepsilon_{0} \varepsilon_{1}}}{\mu c} \nabla \theta^{\varepsilon_{0} \varepsilon_{1}}, \nabla \varphi\right)_{\Omega_{B, B}^{\varepsilon_{0} \varepsilon_{1}}} \\
& +\left(\phi_{\theta}^{\varepsilon_{0} \varepsilon_{1}} \theta_{t}^{\varepsilon_{0} \varepsilon_{1}}, \varphi\right)_{\Omega_{B, B}^{\varepsilon_{\varepsilon} \varepsilon_{1}}}=0 \quad \forall \varphi \in H^{1}\left(\Omega_{B}^{\varepsilon_{0}}\right), \\
\left(\phi_{\theta}^{\varepsilon_{0} \varepsilon_{1}} \theta_{t}^{\varepsilon_{0} \varepsilon_{1}}, \psi\right)_{\Omega_{B, B}^{\varepsilon_{B} \varepsilon_{1}}}+ & \varepsilon_{0}^{2} \varepsilon_{1}^{2}\left(\frac{\mathbf{K}_{\theta}^{\varepsilon_{0} \varepsilon_{1}}}{\mu c} \nabla \theta^{\varepsilon_{0} \varepsilon_{1}}, \nabla \psi\right)_{\Omega_{B, B}^{\varepsilon_{0} \varepsilon_{1}}}=0 \quad \forall \psi \in H_{0}^{1}\left(\Omega_{B, B}^{\varepsilon_{0} \varepsilon_{1}}\right) .
\end{aligned}
$$


Now, let

$$
\beta^{\varepsilon_{0} \varepsilon_{1}}= \begin{cases}\sigma^{\varepsilon_{0} \varepsilon_{1}} & \text { for } x_{0} \in \Omega_{B, F}^{\varepsilon_{0} \varepsilon_{1}}, \\ \theta^{\varepsilon_{0} \varepsilon_{1}} & \text { for } x_{0} \in \Omega_{B, B}^{\varepsilon_{0} \varepsilon_{1}} .\end{cases}
$$

Then, (4.1) is the weak form of

$$
\begin{gathered}
\alpha^{\varepsilon_{0} \varepsilon_{1}} \beta_{t}^{\varepsilon_{0} \varepsilon_{1}}-\nabla \cdot\left(\boldsymbol{\kappa}^{\varepsilon_{0} \varepsilon_{1}} \nabla \beta^{\varepsilon_{0} \varepsilon_{1}}\right)=0 \quad \text { on } \Omega_{B}^{\varepsilon_{0}} \times J, \\
\boldsymbol{\kappa}^{\varepsilon_{0} \varepsilon_{1}} \nabla \beta^{\varepsilon_{0} \varepsilon_{1}} \cdot n_{\Omega_{B}^{\varepsilon_{0}}}=0 \quad \text { on } \partial \Omega_{B}^{\varepsilon_{0}} \times J, \quad \beta^{\varepsilon_{0} \varepsilon_{1}}=\rho_{\text {init }} \quad \text { on } \Omega_{B}^{\varepsilon_{0}} \times\{0\},
\end{gathered}
$$

where

$$
\begin{gathered}
\alpha^{\varepsilon_{0} \varepsilon_{1}}=\chi_{\Omega_{B, F}^{\varepsilon_{0} \varepsilon_{1}}} \phi_{\sigma}^{\varepsilon_{0}}+\chi_{\Omega_{B, B}^{\varepsilon_{0} \varepsilon_{1}}} \phi_{\theta}^{\varepsilon_{0} \varepsilon_{1}} \\
\boldsymbol{\kappa}^{\varepsilon_{0} \varepsilon_{1}}=\varepsilon_{0}^{2} \chi_{\Omega_{B, F}^{\varepsilon_{0} \varepsilon_{1}}} \frac{K_{\sigma}^{\varepsilon_{0}}}{\mu C} \mathbf{I}+\varepsilon_{0}^{2} \varepsilon_{1}^{2} \chi_{\Omega_{B, B}^{\varepsilon_{0} \varepsilon_{1}}} \frac{\mathbf{K}_{\theta}^{\varepsilon_{0} \varepsilon_{1}}}{\mu C} .
\end{gathered}
$$

The initial-boundary value problem (4.4) is a standard parabolic problem with Neumann boundary conditions and it is well known that it has a unique weak solution in $H^{1}\left(J ; L^{2}\left(\Omega_{B}^{\varepsilon_{0}}\right)\right) \cap L^{\infty}\left(J ; H^{1}\left(\Omega_{B}^{\varepsilon_{0}}\right)\right)$. By restrict$\operatorname{ing} \beta^{\varepsilon_{0} \varepsilon_{1}}$, we have the following theorem.

THEOREM 4.1. Assume that $\rho_{\text {init }} \in H^{1}(\Omega)$. Then, for each $\varepsilon_{1}$, there exists a unique solution to the microscopic model posed on $\Omega_{B}^{\varepsilon_{0}} \times J$, and $\sigma^{\varepsilon_{0} \varepsilon_{1}} \in H^{1}(J$; $\left.L^{2}\left(\Omega_{B, F}^{\varepsilon_{0} \varepsilon_{1}}\right)\right) \cap L^{\infty}\left(J ; H^{1}\left(\Omega_{B, F}^{\varepsilon_{0} \varepsilon_{1}}\right)\right)$ and $\theta^{\varepsilon_{0} \varepsilon_{1}} \in H^{1}\left(J ; L^{2}\left(\Omega_{B, B}^{\varepsilon_{0} \varepsilon_{1}}\right)\right) \cap L^{\infty}\left(J ; H^{1}\left(\Omega_{B, B}^{\varepsilon_{0} \varepsilon_{1}}\right)\right)$.

LEMMA 4.2. There exists a constant $C>0$, independent of $\varepsilon_{0}$ and $\varepsilon_{1}$, such that

$$
\begin{gathered}
\left\|\sigma_{t}^{\varepsilon_{0} \varepsilon_{1}}\right\|_{L^{2}\left(J \times \Omega_{B, F}^{\varepsilon_{0} \varepsilon_{1}}\right)}+\left\|\sigma^{\varepsilon_{0} \varepsilon_{1}}\right\|_{L^{\infty}\left(J ; H^{1}\left(\Omega_{B, F}^{\varepsilon_{0} \varepsilon_{1}}\right)\right)} \leq C\left(1+\varepsilon_{0} \varepsilon_{1}\right), \\
\left\|\nabla \theta^{\varepsilon_{0} \varepsilon_{1}}\right\|_{L^{\infty}\left(J ; L^{2}\left(\Omega_{B, B}^{\varepsilon_{0} \varepsilon_{1}}\right)\right)} \leq C\left(1+\left(\varepsilon_{0} \varepsilon_{1}\right)^{-1}\right), \\
\left\|\theta_{t}^{\varepsilon_{0} \varepsilon_{1}}\right\|_{L^{2}\left(J \times \Omega_{B, B}^{\left.\varepsilon_{0} \varepsilon_{1}\right)}\right.}+\left\|\theta^{\varepsilon_{0} \varepsilon_{1}}\right\|_{L^{\infty}\left(J ; L^{2}\left(\Omega_{B, B}^{\varepsilon_{0} \varepsilon_{1}}\right)\right)} \leq C\left(1+\varepsilon_{0} \varepsilon_{1}\right) .
\end{gathered}
$$

Proof. These are the standard parabolic energy estimates for the weak form (4.1) on $\Omega_{B}^{\varepsilon_{0}}$. To derive these estimates, start by taking $\psi=\theta^{\varepsilon_{0} \varepsilon_{1}}$ and then $\psi=\theta_{t}^{\varepsilon_{0} \varepsilon_{1}}$ on a smooth dense subspace.

\section{Homogenization as $\varepsilon_{1} \rightarrow 0$ for fixed $\varepsilon_{0}>0$}

We now begin to find the unique weak solution of the limit problem as $\varepsilon_{1} \rightarrow 0$. Throughout, we use $C$ to denote a generic positive constant that is independent of $\varepsilon_{1}$ and which can be different at different occurrences. 
For fixed $\varepsilon_{0}>0$, it follows from the a priori estimates in Lemma 4.2 that

$$
\begin{aligned}
& \chi_{\Omega_{B, F}^{\varepsilon_{B} \varepsilon_{1}}} \sigma^{\varepsilon_{0} \varepsilon_{1}} \text { is bounded in } H^{1}\left(J ; L^{2}(\Omega)\right), \\
& \chi_{\Omega_{B, F}^{\varepsilon_{0} \varepsilon_{1}}} \frac{K_{\sigma}^{\varepsilon_{0}}}{\mu C} \nabla \sigma^{\varepsilon_{0} \varepsilon_{1}} \text { is bounded in } L^{2}(\Omega \times J), \\
& \chi_{\Omega_{B, B}^{\varepsilon_{0} \varepsilon_{1}}} \theta^{\varepsilon_{0} \varepsilon_{1}} \text { is bounded in } H^{1}\left(J ; L^{2}(\Omega)\right), \\
& \varepsilon_{1} \chi_{\Omega_{B, B}^{\varepsilon_{0} \varepsilon_{1}}} \nabla \theta^{\varepsilon_{0} \varepsilon_{1}} \text { is bounded in } L^{2}(\Omega \times J) .
\end{aligned}
$$

It follows from (5.1) and Lemma 2.1 that

$$
\begin{gathered}
\left(\theta^{\varepsilon_{0} \varepsilon_{1}}\right)^{\sim(2)} \text { is bounded in } L^{2}\left(\Omega \times Y_{0 B} ; H^{1}\left(Y_{1 B} \times J\right)\right), \\
\left(\sigma^{\varepsilon_{0} \varepsilon_{1}}\right)^{\sim(2)} \text { is bounded in } L^{2}\left(\Omega \times Y_{0 B} ; H^{1}\left(Y_{1 F} \times J\right)\right), \\
\left\|\nabla_{X_{2}}\left(\sigma^{\varepsilon_{0} \varepsilon_{1}}\right)^{\sim(2)}\right\|_{L^{2}\left(\Omega \times Y_{0 B} \times Y_{1 F} \times J\right)} \leq\left(C_{\varepsilon_{0}}\right) \varepsilon_{1} .
\end{gathered}
$$

Hence, upon passing to a subsequence in $\varepsilon_{1}$, as $\varepsilon_{1} \rightarrow 0$, the following limits take place weakly in the indicated spaces:

$$
\begin{gathered}
\chi_{\Omega_{B, F}^{\varepsilon_{0} \varepsilon_{1}}} \sigma^{\varepsilon_{0} \varepsilon_{1}} \rightarrow \widehat{\sigma}^{\varepsilon_{0}} \quad \text { in } H^{1}\left(J ; L^{2}(\Omega)\right), \\
\chi_{\Omega_{B, F}^{\varepsilon_{0} \varepsilon_{1}}} \frac{K_{\sigma}^{\varepsilon_{0}}}{\mu C} \nabla \sigma^{\varepsilon_{0} \varepsilon_{1}} \rightarrow \xi^{\varepsilon_{0}} \quad \text { in } L^{2}(\Omega \times J), \\
\left(\sigma^{\varepsilon_{0} \varepsilon_{1}}\right)^{\sim(2)} \rightarrow \sigma^{\varepsilon_{0}} \quad \text { in } L^{2}\left(\Omega \times Y_{0 B} ; H^{1}\left(Y_{1 F} \times J\right)\right), \\
\left(\theta^{\varepsilon_{0} \varepsilon_{1}}\right)^{\sim(2)} \rightarrow \theta^{\varepsilon_{0}} \quad \text { in } L^{2}\left(\Omega \times Y_{0 B} ; H^{1}\left(Y_{1 B} \times J\right)\right) .
\end{gathered}
$$

From (5.4) and the connectedness of $Y_{1 F}, \sigma^{\varepsilon_{0}}$ is independent of $x_{2}$.

LEMMA 5.1. The following relation holds:

$$
\left(\widehat{\sigma}^{\varepsilon_{0}}\right)^{\sim(1)}=\frac{\left|Y_{1 F}\right|}{\left|Y_{1}\right|} \sigma^{\varepsilon_{0}}
$$

Proof. We first note that for $R=B, F$, or blank,

$$
\left(X_{\Omega_{B, R}^{\varepsilon_{0} \varepsilon_{1}}}\right)^{\sim(2)}\left(x_{0}, x_{1}, x_{2}\right)=X_{\Omega \times Y_{0 B} \times Y_{1 R}}\left(x_{0}, x_{1}, x_{2}\right) .
$$


Now, let $\varphi \in C^{\infty}\left(\Omega \times Y_{0 B} \times J\right)$. Then by (5.7),

$$
\begin{aligned}
I^{\varepsilon_{0} \varepsilon_{1}} & =\int_{J \times \Omega \times Y_{0 B} \times Y_{1 F}}\left(\sigma^{\varepsilon_{0} \varepsilon_{1}}\right)^{\sim(2)} \varphi d x_{2} d x_{1} d x_{0} d t \\
& \longrightarrow \int_{J \times \Omega \times Y_{0 B} \times Y_{1 F}} \sigma^{\varepsilon_{0}} \varphi d x_{2} d x_{1} d x_{0} d t \\
& =\left|Y_{1 F}\right| \int_{J \times \Omega \times Y_{0 B}} \sigma^{\varepsilon_{0}} \varphi d x_{1} d x_{0} d t
\end{aligned}
$$

since $\sigma^{\varepsilon_{0}}, \varphi$ do not depend on $x_{2}$.

On the other hand,

$$
\begin{aligned}
I^{\varepsilon_{0} \varepsilon_{1}}= & \left(\left(\sigma^{\varepsilon_{0} \varepsilon_{1}}\right)^{\sim(2)}, X \Omega \times Y_{0 B} \times Y_{1 F} \varphi\right)_{J \times \Omega \times Y_{0 B} \times Y_{1} \times J} \\
= & \left(\left(\chi_{\Omega_{B, F}^{\varepsilon_{0} \varepsilon_{1}}} \sigma^{\varepsilon_{0} \varepsilon_{1}}\right)^{\sim(2)}, \varphi\right)_{\Omega \times Y_{0 B} \times Y_{1} \times J} \\
= & \int_{J \times \Omega_{B}^{\varepsilon_{0}} \times Y_{0} \times Y_{1}} X_{\Omega_{B, F}^{\varepsilon_{0} \varepsilon_{1}}} \sigma^{\varepsilon_{0} \varepsilon_{1}} \varphi\left(\varepsilon_{0} x_{1}+c^{0, \varepsilon_{0}}\left(x_{0}\right), \varepsilon_{1} x_{2}\right. \\
& \left.+c^{1, \varepsilon_{1}}\left(L_{0}\left(x_{0}\right)\right), t\right) d x_{2} d x_{1} d x_{0} d t,
\end{aligned}
$$

where (5.10) and Lemma 2.3 were used.

Now, let $\varepsilon_{1} \rightarrow 0$; by Lemma 2.2,

$$
\begin{aligned}
\varphi\left(\varepsilon_{0} x_{1}+c^{0, \varepsilon_{0}}\left(x_{0}\right), \varepsilon_{1} x_{2}+c^{1, \varepsilon_{1}}\left(L_{0}\left(x_{0}\right)\right), t\right) \\
\longrightarrow \varphi\left(\varepsilon_{0} x_{1}+c^{0, \varepsilon_{0}}\left(x_{0}\right), L_{0}\left(x_{0}\right), t\right)
\end{aligned}
$$

strongly in $L^{2}\left(\Omega_{B}^{\varepsilon_{0}} \times Y_{0} \times Y_{1} \times J\right)$, and this, combined with (5.5), (5.12), and Lemma 2.4, yields

$$
\begin{aligned}
I^{\varepsilon_{0} \varepsilon_{1}} & \longrightarrow \int_{J \times \Omega_{B}^{\varepsilon_{0} \times Y_{0} \times Y_{1}}} \widehat{\sigma}^{\varepsilon_{0}}\left(x_{0}, t\right) \varphi\left(\varepsilon_{0} x_{1}+c^{0, \varepsilon_{0}}\left(x_{0}\right), L_{0}\left(x_{0}\right), t\right) d x_{2} d x_{1} d x_{0} d t \\
& =\left|Y_{1}\right| \int_{J \times \Omega_{B}^{\varepsilon_{0}} \times Y_{0}} \widehat{\sigma}^{\varepsilon_{0}}\left(x_{0}, t\right) \varphi\left(\varepsilon_{0} x_{1}+c^{0, \varepsilon_{0}}\left(x_{0}\right), L_{0}\left(x_{0}\right), t\right) d x_{1} d x_{0} d t \\
& =\left|Y_{1}\right| \int_{J \times \Omega \times Y_{0 B}}\left(\widehat{\sigma}^{\varepsilon_{0}}\right)^{\sim(1)}\left(x_{0}, x_{1}, t\right) \varphi\left(x_{0}, x_{1}, t\right) d x_{1} d x_{0} d t .
\end{aligned}
$$

Since $\varphi$ is arbitrary, (5.11) and (5.14) imply the lemma. 
We now derive an equation satisfied by $\theta^{\varepsilon_{0}}$. Let $\psi \in L^{2}\left(\Omega \times Y_{0 B} \times J\right.$; $\left.H_{0}^{1}\left(Y_{1 B}\right)\right)$. Set

$$
\begin{aligned}
& \widehat{\psi}\left(x_{0}, x_{1}, z, t\right) \\
& =\left\{\begin{array}{c}
\psi\left(x_{0}, x_{1}, \frac{z-\varepsilon_{0} c^{1, \varepsilon_{1}}\left(x_{1}\right)-c^{0, \varepsilon_{0}}\left(x_{0}\right)}{\varepsilon_{0} \varepsilon_{1}}, t\right) \\
\quad \text { if } z \in \varepsilon_{0} \varepsilon_{1} Y_{1 B}+\varepsilon_{0} c^{1, \varepsilon_{1}}\left(x_{1}\right)+c^{0, \varepsilon_{0}}\left(x_{0}\right), \\
0 \quad \text { elsewhere. }
\end{array}\right.
\end{aligned}
$$

Then $\widehat{\psi} \in L^{2}\left(\Omega \times Y_{0 B} \times J ; H_{0}^{1}\left(\varepsilon_{0} \varepsilon_{1} Y_{1 B}+\varepsilon_{0} c^{1, \varepsilon_{1}}\left(x_{1}\right)+c^{0, \varepsilon_{0}}\left(x_{0}\right)\right)\right)$. For fixed $\left(x_{0}, x_{1}\right) \in \Omega \times Y_{0 B}$, use $\widehat{\psi}$ as a test function in (4.2) to obtain

$$
\begin{aligned}
\int_{\varepsilon_{0} \varepsilon_{1} Y_{1 B}+\varepsilon_{0} c^{1, \varepsilon_{1}}\left(x_{1}\right)+c^{0, \varepsilon_{0}}\left(x_{0}\right)} & \phi_{\theta}^{\varepsilon_{0} \varepsilon_{1}}(z) \theta_{t}^{\varepsilon_{0} \varepsilon_{1}}(z) \psi \\
\times\left(x_{0},\right. & \left.x_{1}, \frac{z-\varepsilon_{0} c^{1, \varepsilon_{1}}\left(x_{1}\right)-c^{0, \varepsilon_{0}}\left(x_{0}\right)}{\varepsilon_{0} \varepsilon_{1}}, t\right) d z \\
+\varepsilon_{0}^{2} \varepsilon_{1}^{2} \int_{\varepsilon_{0} \varepsilon_{1} Y_{1 B}+\varepsilon_{0} c^{1, \varepsilon_{1}}\left(x_{1}\right)+c^{0, \varepsilon_{0}}\left(x_{0}\right)} \frac{\mathbf{K}_{\theta}^{\varepsilon_{0} \varepsilon_{1}}}{\mu c}(z) \nabla_{z} \theta^{\varepsilon_{0} \varepsilon_{1}}(z) \cdot \nabla_{z} \psi & \\
& \times\left(x_{0}, x_{1}, \frac{z-\varepsilon_{0} c^{1, \varepsilon_{1}}\left(x_{1}\right)-c^{0, \varepsilon_{0}}\left(x_{0}\right)}{\varepsilon_{0} \varepsilon_{1}}, t\right) d z=0 .
\end{aligned}
$$

Use the dilation $z \mapsto \varepsilon_{0} \varepsilon_{1} x_{2}+\varepsilon_{0} c^{1, \varepsilon_{1}}\left(x_{1}\right)+c^{0, \varepsilon_{0}}\left(x_{0}\right)$ and integrate over $\Omega \times$ $Y_{0 B} \times J$ to get

$$
\begin{aligned}
& \int_{J \times \Omega \times Y_{0 B} \times Y_{1 B}} \phi_{\theta}\left(x_{2}\right)\left(\theta_{t}^{\varepsilon_{0} \varepsilon_{1}}\right)^{\sim(2)} \psi\left(x_{0}, x_{1}, x_{2}, t\right) d x_{2} d x_{1} d x_{0} d t \\
& +\int_{J \times \Omega \times Y_{0 B} \times Y_{1 B}} \frac{\mathbf{K}_{\theta}}{\mu C}\left(x_{2}\right) \nabla_{x_{2}}\left(\theta^{\varepsilon_{0} \varepsilon_{1}}\right)^{\sim(2)} \cdot \nabla_{x_{2}} \psi\left(x_{0}, x_{1}, x_{2}, t\right) d x_{2} d x_{1} d x_{0} d t=0,
\end{aligned}
$$

where we used

$$
\left(\phi_{\theta}^{\varepsilon_{0} \varepsilon_{1}}\right)^{\sim(2)}=\chi_{\Omega \times Y_{0 B} \times Y_{1 B}} \phi_{\theta}, \quad\left(\frac{\mathbf{K}_{\theta}^{\varepsilon_{0} \varepsilon_{1}}}{\mu C}\right)^{\sim(2)}=\chi_{\Omega \times Y_{0 B} \times Y_{1 B}} \frac{\mathbf{K}_{\theta}}{\mu C} .
$$

Next, let $\varepsilon_{1} \rightarrow 0$ and use the weak limits to get

$$
\left(\phi_{\theta} \theta_{t}^{\varepsilon_{0}}, \psi\right)_{\Omega \times Y_{0 B} \times Y_{1 B} \times J}+\left(\frac{\mathbf{K}_{\theta}}{\mu c} \nabla_{x_{2}} \theta^{\varepsilon_{0}}, \nabla_{x_{2}} \psi\right)_{\Omega \times Y_{0 B} \times Y_{1 B} \times J}=0
$$


that is, $\theta^{\varepsilon_{0}}$ is a weak solution of

$$
\phi_{\theta} \theta_{t}^{\varepsilon_{0}}-\nabla_{x_{2}} \cdot\left(\frac{\mathbf{K}_{\theta}}{\mu c} \nabla_{x_{2}} \theta^{\varepsilon_{0}}\right)=0
$$

in $L^{2}\left(\Omega \times Y_{0 B} ; H^{1}\left(Y_{1 B} \times J\right)\right)$.

We now begin to derive an equation for $\widehat{\sigma}^{\varepsilon_{0}}$. Let $\varphi \in L^{2}\left(J ; H^{1}\left(\Omega_{B}^{\varepsilon_{0}}\right)\right)$ and integrate (4.1) over $J$ to get

$$
\begin{aligned}
\left(\phi_{\sigma}^{\varepsilon_{0}} \sigma_{t}^{\varepsilon_{0} \varepsilon_{1}}, \varphi\right)_{J \times \Omega_{B, F}^{\varepsilon_{0} \varepsilon_{1}}}+\varepsilon_{0}^{2}\left(\frac{K_{\sigma}^{\varepsilon_{0}}}{\mu c} \nabla \sigma^{\varepsilon_{0} \varepsilon_{1}}, \varphi\right)_{J \times \Omega_{B, F}^{\varepsilon_{0} \varepsilon_{1}}} \\
\quad+\varepsilon_{0}^{2} \varepsilon_{1}^{2}\left(\frac{\mathbf{K}_{\theta}^{\varepsilon_{0} \varepsilon_{1}}}{\mu c} \nabla \theta^{\varepsilon_{0} \varepsilon_{1}}, \nabla \varphi\right)_{J \times \Omega_{B, B}^{\varepsilon_{0} \varepsilon_{1}}}+\left(\phi_{\theta}^{\varepsilon_{0} \varepsilon_{1}} \theta_{t}^{\varepsilon_{0} \varepsilon_{1}}, \varphi\right)_{J \times \Omega_{B, B}^{\varepsilon_{0} \varepsilon_{1}}} \\
=T_{1}+T_{2}+T_{3}+T_{4}=0 .
\end{aligned}
$$

We now let $\varepsilon_{1} \rightarrow 0$;

$$
\begin{aligned}
& T_{1} \longrightarrow \int_{J \times \Omega_{B}^{\varepsilon_{0}}} \phi_{\sigma}^{\varepsilon_{0}} \widehat{\sigma}_{t}^{\varepsilon_{0}} \varphi d x_{0} d t \quad \text { by }(5.5), \\
& T_{2} \longrightarrow \varepsilon_{0}^{2} \int_{J \times \Omega_{B}^{\varepsilon_{0}}} \xi^{\varepsilon_{0}} \cdot \nabla \varphi d x_{0} d t \quad \text { by (5.6), }
\end{aligned}
$$

$T_{3} \longrightarrow 0$ since the term is bounded by a multiple of $\varepsilon_{1}^{2}$.

We now investigate the convergence of $T_{4}$. By Lemmas 2.1, 2.2, (5.8), and Lemma 2.4, we have

$$
\begin{aligned}
T_{4}= & \left(\left|Y_{0}\right|\left|Y_{1}\right|\right)^{-1}\left(\left(\phi_{\theta}^{\varepsilon_{0} \varepsilon_{1}}\right)^{\sim(2)}\left(\theta_{t}^{\varepsilon_{0} \varepsilon_{1}}\right)^{\sim(2)}, \varphi^{\sim(2)}\right)_{J \times \Omega \times Y_{0 B} \times Y_{1 B}} \\
= & \left(\left|Y_{0}\right|\left|Y_{1}\right|\right)^{-1}\left(\phi_{\theta}\left(\theta_{t}^{\varepsilon_{0} \varepsilon_{1}}\right)^{\sim(2)}, \varphi^{\sim(2)}\right)_{J \times \Omega \times Y_{0 B} \times Y_{1 B}} \\
\longrightarrow\left(\left|Y_{0}\right|\left|Y_{1}\right|\right)^{-1}\left(\phi_{\theta} \theta_{t}^{\varepsilon_{0}}, \varphi^{\sim(1)}\right)_{J \times \Omega \times Y_{0 B} \times Y_{1 B}} & \\
= & \left(\left|Y_{0}\right|\left|Y_{1}\right|\right)^{-1} \int_{J \times \Omega_{B}^{\varepsilon_{0}} \times Y_{0} \times Y_{1 B}} \phi_{\theta} \theta_{t}^{\varepsilon_{0}}\left(\varepsilon_{0} x_{1}+c^{0, \varepsilon_{0}}\left(x_{0}\right), L_{0}\left(x_{0}\right), x_{2}, t\right) \\
\times & \times \varphi\left(x_{0}, t\right) d x_{2} d x_{1} d x_{0} d t .
\end{aligned}
$$


It follows from (5.22) and (5.23) that

$$
\begin{aligned}
& \int_{J \times \Omega_{B}^{\varepsilon_{0}}} \phi_{\sigma}^{\varepsilon_{0}} \widehat{\sigma}_{t}^{\varepsilon_{0}} \varphi d x_{0} d t+\varepsilon_{0}^{2} \int_{J \times \Omega_{B}^{\varepsilon_{0}}} \xi^{\varepsilon_{0}} \cdot \nabla \varphi d x_{0} d t \\
& +\left(\left|Y_{0}\right|\left|Y_{1}\right|\right)^{-1} \int_{J \times \Omega_{B}^{\varepsilon_{0}} \times Y_{0} \times Y_{1 B}} \phi_{\theta} \theta_{t}^{\varepsilon_{0}}\left(\varepsilon_{0} x_{1}+c^{0, \varepsilon_{0}}\left(x_{0}\right), L_{0}\left(x_{0}\right), x_{2}, t\right) \\
& \quad \times \varphi\left(x_{0}, t\right) \phi_{\theta}\left(x_{2}\right) d x_{2} d x_{1} d x_{0} d t \\
& =0 \quad \forall \varphi \in L^{2}\left(J ; H^{1}(\Omega)\right) .
\end{aligned}
$$

We now relate $\xi^{\varepsilon_{0}}$ to $\widehat{\sigma}^{\varepsilon_{0}}$. For $j=1,2,3$, let $\omega_{j}=\omega_{j}\left(x_{2}\right)$ be the $Y_{1}$-periodic solution, modulo constants, to the Neumann problem

$$
\Delta_{x_{2}} \omega_{j}=0 \quad \text { in } Y_{1 F}, \quad \nabla_{x_{2}} \omega_{j} \cdot v=-\mathbf{e}_{j} \cdot v=-v_{j} \quad \text { on } \partial Y_{1 B},
$$

where $v$ is the outer unit normal to $\partial Y_{1 B}$. Define $\omega_{j}^{\varepsilon_{0} \varepsilon_{1}} \in H^{1}\left(\Omega_{B}^{\varepsilon_{0}}\right)$ by

$$
\omega_{j}^{\varepsilon_{0} \varepsilon_{1}}\left(x_{0}\right)=\varepsilon_{0} \varepsilon_{1} \varepsilon \omega_{j}\left(L_{1}\left(x_{0}\right)\right)
$$

where $\varepsilon: H^{1}\left(Y_{1 F}\right) \rightarrow H^{1}\left(Y_{1}\right)$ is a bounded extension operator [12].

Hence $\left(\omega_{j}^{\varepsilon_{0} \varepsilon_{1}}\right)^{\sim(2)}\left(x_{0}, x_{1}, x_{2}\right)=\varepsilon_{0} \varepsilon_{1}\left(\varepsilon \omega_{j}\right)\left(x_{2}\right)$. A similar argument shows that

$$
\left(\nabla_{x_{0}} \omega_{j}^{\varepsilon_{0} \varepsilon_{1}}\right)^{\sim(2)}\left(x_{0}, x_{1}, x_{2}\right)=\left(\nabla_{x_{2}} \varepsilon \omega_{j}\right)\left(x_{2}\right)
$$

Now let

$$
\omega_{i j}=\frac{1}{\left|Y_{1}\right|} \int_{Y_{1 F}} \partial_{i} \omega_{j}\left(x_{2}\right) d x_{2}
$$

where $\partial_{i}=\partial / \partial x_{2, i}$.

An argument as in the proof of [5, Lemma 4.3] can be used to verify the following lemma.

LEMMA 5.2. As $\varepsilon_{1} \rightarrow 0$,

$$
\begin{gathered}
\omega_{j}^{\varepsilon_{0} \varepsilon_{1}} \longrightarrow 0 \text { strongly in } L^{2}\left(\Omega_{B}^{\varepsilon_{0}}\right), \\
\varepsilon_{0} \varepsilon_{1} \nabla \omega_{j}^{\varepsilon_{0} \varepsilon_{1}} \longrightarrow 0 \text { strongly in } L^{2}\left(\Omega_{B}^{\varepsilon_{0}}\right), \\
\chi_{\Omega_{B, F}^{\varepsilon_{0} \varepsilon_{1}} \partial_{i} \omega_{j}^{\varepsilon_{0} \varepsilon_{1}}} \longrightarrow \omega_{i j} \text { weakly in } L^{2}\left(\Omega_{B}^{\varepsilon_{0}}\right) .
\end{gathered}
$$


342 Analysis of a multiple-porosity model

If $\psi \in H^{1}\left(\Omega_{B, F}^{\varepsilon_{0} \varepsilon_{1}}\right)$, then

$$
\left(\nabla \omega_{j}^{\varepsilon_{0} \varepsilon_{1}}+\mathbf{e}_{j}, \nabla \psi\right)_{\Omega_{B, F}^{\varepsilon_{0} \varepsilon_{1}}}=0
$$

since $\omega_{j}$ solves the above Neumann problem. Now, for $\varphi \in C^{\infty}\left(\left(\Omega_{B}^{\varepsilon_{0}} \times\right.\right.$ $\left.J)^{-}\right)$, take $\psi=\sigma^{\varepsilon_{0} \varepsilon_{1}}\left(K_{\sigma}^{\varepsilon_{0}} / \mu c\right) \varphi$ in (5.30) and integrate in time to get

$$
\begin{aligned}
0= & \left(\nabla \omega_{j}^{\varepsilon_{0} \varepsilon_{1}}, \frac{K_{\sigma}^{\varepsilon_{0}}}{\mu c} \varphi \nabla\left(\sigma^{\varepsilon_{0} \varepsilon_{1}}\right)\right)_{J \times \Omega_{B, F}^{\varepsilon_{0} \varepsilon_{1}}}+\left(\nabla \omega_{j}^{\varepsilon_{0} \varepsilon_{1}}, \sigma^{\varepsilon_{0} \varepsilon_{1}} \nabla\left(\frac{K_{\sigma}^{\varepsilon_{0}}}{\mu c} \varphi\right)\right)_{J \times \Omega_{B, F}^{\varepsilon_{0} \varepsilon_{1}}} \\
& +\left(\mathbf{e}_{j}, \frac{K_{\sigma}^{\varepsilon_{0}}}{\mu c} \varphi \nabla\left(\sigma^{\varepsilon_{0} \varepsilon_{1}}\right)\right)_{J \times \Omega_{B, F}^{\varepsilon_{0} \varepsilon_{1}}}+\left(\mathbf{e}_{j}, \sigma^{\varepsilon_{0} \varepsilon_{1}} \nabla\left(\frac{K_{\sigma}^{\varepsilon_{0}}}{\mu c} \varphi\right)\right)_{J \times \Omega_{B, F}^{\varepsilon_{0} \varepsilon_{1}}} \\
= & T_{5}+T_{6}+T_{7}+T_{8} .
\end{aligned}
$$

We proceed to let $\varepsilon_{1} \rightarrow 0$ in each term of (5.31). We begin with term $T_{5}$. Use $\omega_{j}^{\varepsilon_{0} \varepsilon_{1}} \varphi$ as a test function in (4.1) to obtain

$$
\begin{aligned}
\varepsilon_{0}^{2}\left(\frac{K_{\sigma}^{\varepsilon_{0}}}{\mu c} \nabla \sigma^{\varepsilon_{0} \varepsilon_{1}}, \varphi \nabla\left(\omega_{j}^{\varepsilon_{0} \varepsilon_{1}}\right)\right)_{\Omega_{B, F}^{\varepsilon_{0} \varepsilon_{1}}} \\
=-\left(\phi_{\sigma}^{\varepsilon_{0}} \sigma_{t}^{\varepsilon_{0} \varepsilon_{1}}, \omega_{j}^{\varepsilon_{0} \varepsilon_{1}} \varphi\right)_{\Omega_{B, F}^{\varepsilon_{0} \varepsilon_{1}}}-\varepsilon_{0}^{2} \varepsilon_{1}^{2}\left(\frac{\mathbf{K}_{\theta}^{\varepsilon_{0} \varepsilon_{1}}}{\mu c} \nabla \theta^{\varepsilon_{0} \varepsilon_{1}}, \omega_{j}^{\varepsilon_{0} \varepsilon_{1}} \nabla(\varphi)\right)_{\Omega_{B, B}^{\varepsilon_{0} \varepsilon_{1}}} \\
\quad-\varepsilon_{0}^{2} \varepsilon_{1}^{2}\left(\frac{\mathbf{K}_{\theta}^{\varepsilon_{0} \varepsilon_{1}}}{\mu c} \nabla \theta^{\varepsilon_{0} \varepsilon_{1}}, \varphi \nabla\left(\omega_{j}^{\varepsilon_{0} \varepsilon_{1}}\right)\right)_{\Omega_{B, B}^{\varepsilon_{0} \varepsilon_{1}}}-\left(\phi_{\theta}^{\varepsilon_{0} \varepsilon_{1}} \theta_{t}^{\varepsilon_{0} \varepsilon_{1}}, \omega_{j}^{\varepsilon_{0} \varepsilon_{1}} \varphi\right)_{\Omega_{B, B}^{\varepsilon_{0} \varepsilon_{1}}} \\
\quad-\varepsilon_{0}^{2}\left(\frac{K_{\sigma}^{\varepsilon_{0}}}{\mu c} \nabla \sigma^{\varepsilon_{0} \varepsilon_{1}}, \omega_{j}^{\varepsilon_{0} \varepsilon_{1}} \nabla(\varphi)\right)_{\Omega_{B, F}^{\varepsilon_{0} \varepsilon_{1}}}
\end{aligned}
$$

It follows from (5.1), Lemma 5.2, and the boundedness of $\nabla \omega_{j}^{\varepsilon_{0} \varepsilon_{1}}$ that

$$
T_{5} \longrightarrow 0 \quad \text { as } \varepsilon_{1} \longrightarrow 0
$$

For term $T_{6}$, we have

$$
\begin{aligned}
T_{6} & =\left(\left|Y_{0}\right|\left|Y_{1}\right|\right)^{-1}\left(\nabla_{x_{2}} \omega_{j},\left(\sigma^{\varepsilon_{0} \varepsilon_{1}}\right)^{\sim(2)}\left[\nabla_{x_{0}}\left(\frac{K_{\sigma}^{\varepsilon_{0}}}{\mu c} \varphi\right)\right]^{\sim(2)}\right)_{J \times \Omega \times Y_{0 B} \times Y_{1 F}} \\
& \stackrel{\varepsilon_{1} \rightarrow 0}{\longrightarrow}\left(\left|Y_{0}\right|\left|Y_{1}\right|\right)^{-1}\left(\nabla_{x_{2}} \omega_{j}, \sigma^{\varepsilon_{0}}\left[\nabla_{x_{0}}\left(\frac{K_{\sigma}^{\varepsilon_{0}}}{\mu c} \varphi\right)\right]^{\sim(1)}\right)_{J \times \Omega \times Y_{0 B} \times Y_{1 F}}
\end{aligned}
$$




$$
\begin{array}{r}
=\left(\left|Y_{0}\right|\left|Y_{1}\right|\right)^{-1} \int_{J \times \Omega \times Y_{0 B} \times Y_{1 F}} \sigma^{\varepsilon_{0}}\left(\varepsilon_{0} x_{1}+c^{0, \varepsilon_{0}}\left(x_{0}\right), L_{0}\left(x_{0}\right), t\right) \\
\times \nabla_{x_{2}} \omega_{j} \cdot \nabla_{x_{0}}\left(\frac{K_{\sigma}^{\varepsilon_{0}}}{\mu c} \varphi\right) d x_{2} d x_{1} d x_{0} d t \\
=\left(\left|Y_{0}\right|\right)^{-1} \int_{J \times \Omega \times Y_{0 B}} X_{\Omega_{B}^{\varepsilon_{0}}} \sigma^{\varepsilon_{0}}\left(\varepsilon_{0} x_{1}+c^{0, \varepsilon_{0}}\left(x_{0}\right), L_{0}\left(x_{0}\right), t\right) \\
\times\left(\sum_{i} \omega_{i j} \partial_{i}\left(\frac{K_{\sigma}^{\varepsilon_{0}}}{\mu c} \varphi\right)\left(x_{0}\right)\right) d x_{1} d x_{0} d t
\end{array}
$$

by Lemma 2.1, (5.7), Lemmas 2.2, 2.4, and the definition of $\omega_{i j}$.

But we observe from Lemma 5.1 that

$$
\sigma^{\varepsilon_{0}}\left(\varepsilon_{0} x_{1}+c^{0, \varepsilon_{0}}\left(x_{0}\right), L_{0}\left(x_{0}\right), t\right)=\frac{\left|Y_{1}\right|}{\left|Y_{1 F}\right|} \widehat{\sigma}^{\varepsilon_{0}}\left(x_{0}, t\right) .
$$

Hence

$$
\begin{aligned}
T_{6} \stackrel{\varepsilon_{1} \rightarrow 0}{\longrightarrow} 0 \frac{\left|Y_{1}\right|}{\left|Y_{0}\right|\left|Y_{1 F}\right|} \int_{J \times \Omega \times Y_{0}} & X_{\Omega_{B}^{\varepsilon_{0}}}\left(x_{0}\right) \widehat{\sigma}^{\varepsilon_{0}}\left(x_{0}, t\right) \\
\times & \left(\sum_{i} \omega_{i j} \partial_{i}\left(\frac{K_{\sigma}^{\varepsilon_{0}}}{\mu c} \varphi\left(x_{0}\right)\right)\right) d x_{0} d t .
\end{aligned}
$$

For the term $T_{8}$, we have by (5.5)

$$
\begin{aligned}
T_{8} & \stackrel{\varepsilon_{1} \rightarrow 0}{\longrightarrow}\left(\mathbf{e}_{j}, \widehat{\sigma}^{\varepsilon_{0}} \nabla\left(\frac{K_{\sigma}^{\varepsilon_{0}}}{\mu c} \varphi\right)\right)_{J \times \Omega_{B}^{\varepsilon_{0}}} \\
& =\int_{J \times \Omega_{B}^{\varepsilon_{0}}} \widehat{\sigma}^{\varepsilon_{0}}\left(x_{0}, t\right)\left(\sum_{i} \delta_{i j} \partial_{i}\left(\frac{K_{\sigma}^{\varepsilon_{0}}}{\mu c} \varphi\left(x_{0}\right)\right)\right) d x_{0} d t .
\end{aligned}
$$

Hence, by (5.6), (5.31), (5.33), (5.36), and (5.37),

$$
\begin{aligned}
& T_{7} \stackrel{\varepsilon_{1} \rightarrow 0}{\longrightarrow}\left(\xi_{j}^{\varepsilon_{0}}, \varphi\right)_{J \times \Omega_{B}^{\varepsilon_{0}}} \\
& \quad=\int_{J \times \Omega_{B}^{\varepsilon_{0}}} \sum_{i}\left(\frac{\left|Y_{1}\right|}{\left|Y_{1 F}\right|} \omega_{i j}+\delta_{i j}\right) \partial_{i}\left(\widehat{\sigma}^{\varepsilon_{0}}\left(x_{0}, t\right)\right) \frac{K_{\sigma}^{\varepsilon_{0}}}{\mu c} \varphi\left(x_{0}\right) d x_{0} d t .
\end{aligned}
$$

So, define

$$
\left(\mathbf{K}_{\sigma}^{h \varepsilon_{0}}\right)_{i j} \equiv K_{\sigma}^{\varepsilon_{0}}\left(\omega_{i j}+\frac{\left|Y_{1 F}\right|}{\left|Y_{1}\right|} \delta_{i j}\right) .
$$


344 Analysis of a multiple-porosity model

Then, as in [5], $\mathbf{K}_{\sigma}^{h \varepsilon_{0}}$ is a bounded, symmetric, positive-definite tensor. Then we can write the equality in (5.38) as

$$
\left(\xi^{\varepsilon_{0}}, \varphi\right)_{J \times \Omega_{B}^{\varepsilon_{0}}}=\left(\frac{\left|Y_{1}\right|}{\left|Y_{1 F}\right|} \frac{\mathbf{K}_{\sigma}^{h \varepsilon_{0}}}{\mu c} \nabla \widehat{\sigma}^{\varepsilon_{0}}, \varphi\right)_{J \times \Omega_{B}^{\varepsilon_{0}}},
$$

that is,

$$
\xi^{\varepsilon_{0}}=\frac{\left|Y_{1}\right|}{\left|Y_{1 F}\right|} \frac{\mathbf{K}_{\sigma}^{h \varepsilon_{0}}}{\mu c} \nabla \widehat{\sigma}^{\varepsilon_{0}} \quad \text { in } \Omega_{B}^{\varepsilon_{0}} \times J
$$

Next define

$$
r^{\varepsilon_{0}} \equiv \frac{\left|Y_{1}\right|}{\left|Y_{1 F}\right|} \widehat{\sigma}^{\varepsilon_{0}}, \quad \phi_{\sigma}^{h \varepsilon_{0}} \equiv \frac{\left|Y_{1 F}\right|}{\left|Y_{1}\right|} \phi_{\sigma}^{\varepsilon_{0}}
$$

Then we can rewrite (5.24) as

$$
\begin{gathered}
\int_{J \times \Omega_{B}^{\varepsilon_{0}}} \phi_{\sigma}^{h \varepsilon_{0}} r_{t}^{\varepsilon_{0}} \varphi d x_{0} d t+\varepsilon_{0}^{2} \int_{J \times \Omega_{B}^{\varepsilon_{0}}} \frac{\mathbf{K}_{\sigma}^{h \varepsilon_{0}}}{\mu c} \nabla r^{\varepsilon_{0}} \cdot \nabla \varphi d x_{0} d t \\
+\left(\left|Y_{0}\right|\left|Y_{1}\right|\right)^{-1} \int_{J \times \Omega_{B}^{\varepsilon_{0}} \times Y_{0} \times Y_{1 B}} \phi_{\theta}\left(x_{2}\right) \theta_{t}^{\varepsilon_{0}}\left(\varepsilon_{0} x_{1}+c^{0, \varepsilon_{0}}\left(x_{0}\right), L_{0}\left(x_{0}\right), x_{2}, t\right) \\
\quad \times \varphi\left(x_{0}, t\right) d x_{2} d x_{1} d x_{0} d t \\
=0 \quad \forall \varphi \in L^{2}\left(J ; H^{1}\left(\Omega_{B}^{\varepsilon_{0}}\right)\right),
\end{gathered}
$$

which is a weak form of the following partial differential equation:

$$
\phi_{\sigma}^{h \varepsilon_{0}} r_{t}^{\varepsilon_{0}}+\varepsilon_{0}^{2} \nabla \cdot\left(\frac{\mathbf{K}_{\sigma}^{h \varepsilon_{0}}}{\mu c} \nabla r^{\varepsilon_{0}}\right)+f_{B, B}^{\varepsilon_{0}}=0 \quad \text { in } \Omega_{B}^{\varepsilon_{0}} \times J,
$$

where

$f_{B, B}^{\varepsilon_{0}}=\left(\left|Y_{0} \| Y_{1}\right|\right)^{-1} \int_{Y_{0} \times Y_{1 B}} \phi_{\theta}\left(x_{2}\right) \theta_{t}^{\varepsilon_{0}}\left(\varepsilon_{0} x_{1}+c^{0, \varepsilon_{0}}\left(x_{0}\right), L_{0}\left(x_{0}\right), x_{2}, t\right) d x_{2} d x_{1}$.

We now determine the initial and boundary conditions for $\theta^{\varepsilon_{0}}$ and $\widehat{\sigma}^{\varepsilon_{0}}$. We begin with the following lemma, which can be established by means of Lemmas 2.1, 2.2, and 2.4. 
LEMMA 5.3. The following is true:

$$
\chi_{\Omega_{B, F}^{\varepsilon_{0} \varepsilon_{1}}} \stackrel{\varepsilon_{1} \rightarrow 0}{\longrightarrow} \frac{\left|Y_{1 F}\right|}{\left|Y_{1}\right|} \text { weak } \text { win }^{\infty}\left(\Omega_{B}^{\varepsilon_{0}}\right) .
$$

By Lemma 5.3,

$$
\chi_{\Omega_{B, F}^{\varepsilon_{0} \varepsilon_{1}}} \rho_{\text {init }} \longrightarrow \frac{\left|Y_{1 F}\right|}{\left|Y_{1}\right|} \rho_{\text {init }}
$$

weakly in $L^{2}\left(\Omega_{B}^{\varepsilon_{0}}\right)$ as $\varepsilon_{1} \rightarrow 0$. Also, by (5.5) and weak continuity of the appropriate trace map,

$$
\chi_{\Omega_{B, F}^{\varepsilon_{0} \varepsilon_{1}}} \sigma^{\varepsilon_{0} \varepsilon_{1}}\left(x_{0}, 0\right) \longrightarrow \widehat{\sigma}^{\varepsilon_{0}}\left(x_{0}, 0\right)
$$

weakly in $L^{2}\left(\Omega_{B}^{\varepsilon_{0}}\right)$ as $\varepsilon_{1} \rightarrow 0$. Therefore, since $\sigma^{\varepsilon_{0} \varepsilon_{1}}\left(x_{0}, 0\right)=\rho_{\text {init }}\left(x_{0}\right)$, it must be true that

$$
\frac{\left|Y_{1 F}\right|}{\left|Y_{1}\right|} \rho_{\text {init }}=\widehat{\sigma}^{\varepsilon_{0}} \quad \text { in } \Omega_{B}^{\varepsilon_{0}} \times\{0\} .
$$

A more convenient way of writing this is

$$
r^{\varepsilon_{0}}=\rho_{\text {init }} \quad \text { in } \Omega_{B}^{\varepsilon_{0}} \times\{0\} .
$$

To obtain the initial condition for $\theta^{\varepsilon_{0}}$, let $\tau_{0}: H^{1}\left(Y_{1 B} \times J\right) \rightarrow H^{1 / 2}\left(Y_{1 B} \times\right.$ $\{0\})$ denote the trace map. Then, as $\varepsilon_{1} \rightarrow 0$,

$$
\tau_{0}\left(\left(\theta^{\varepsilon_{0} \varepsilon_{1}}\right)^{\sim(2)}\right) \rightarrow \tau_{0}\left(\theta^{\varepsilon_{0}}\right)
$$

weakly in $L^{2}\left(\Omega \times Y_{0 B} \times Y_{1 B}\right)$. But

$$
\tau_{0}\left(\left(\theta^{\varepsilon_{0} \varepsilon_{1}}\right)^{\sim(2)}\right)=\left(\rho_{\text {init }}\right)^{\sim(2)} \forall \varepsilon_{0}, \varepsilon_{1} .
$$

Also, by Lemma 2.2, $\left(\rho_{\text {init }}\right)^{\sim(2)} \rightarrow\left(\rho_{\text {init }}\right)^{\sim(1)}$ strongly as $\varepsilon_{1} \rightarrow 0$. Hence

$$
\theta^{\varepsilon_{0}}=\tilde{\rho_{\text {init }}^{(1)}} \text { in } \Omega \times Y_{0 B} \times \Upsilon_{1 B} \times\{0\} .
$$

To obtain a boundary condition on $\partial Y_{1 B}$, for $\theta^{\varepsilon_{0}}$, let $\tau: H^{1}\left(Y_{1 B}\right) \rightarrow$ $H^{1 / 2}\left(\partial Y_{1 B} \times J\right)$ denote the trace map. Then

$$
\begin{aligned}
& \tau\left(\left(\sigma^{\varepsilon_{0} \varepsilon_{1}}\right)^{\sim(2)}\right) \longrightarrow \tau\left(\sigma^{\varepsilon_{0}}\right)=\frac{\left|Y_{1}\right|}{\left|Y_{1 F}\right|} \tau\left(\left(\widehat{\sigma}^{\varepsilon_{0}}\right)^{\sim(1)}\right), \\
& \tau\left(\left(\theta^{\varepsilon_{0} \varepsilon_{1}}\right)^{\sim(2)}\right) \longrightarrow \tau\left(\theta^{\varepsilon_{0}}\right)
\end{aligned}
$$


346 Analysis of a multiple-porosity model

weakly in $L^{2}\left(\Omega \times Y_{0 B} \times \partial Y_{1 B} \times J\right)$. Since, from the boundary condition in (3.2),

$$
\tau\left(\left(\sigma^{\varepsilon_{0} \varepsilon_{1}}\right)^{\sim(2)}\right)=\tau\left(\left(\theta^{\varepsilon_{0} \varepsilon_{1}}\right)^{\sim(2)}\right)
$$

it follows that

$$
\tau\left(\theta^{\varepsilon_{0}}\right)=\frac{\left|Y_{1}\right|}{\left|Y_{1 F}\right|} \tau\left(\left(\widehat{\sigma}^{\varepsilon_{0}}\right)^{\sim(1)}\right)
$$

that is,

$$
\theta^{\varepsilon_{0}}=\frac{\left|Y_{1}\right|}{\left|Y_{1 F}\right|}\left(\widehat{\sigma}^{\varepsilon_{0}}\right)^{\sim(1)} \equiv\left(r^{\varepsilon_{0}}\right)^{\sim(1)} \quad \text { on } \Omega \times Y_{0 B} \times \partial Y_{1 B} \times J
$$

Thus, $\theta^{\varepsilon_{0}}$ is a solution of problems (5.20), (5.57), and (5.53) and $r^{\varepsilon_{0}}$ is a solution of problems (5.44) and (5.50) with the boundary condition

$$
\frac{\mathbf{K}_{\sigma}^{h \varepsilon_{0}}}{\mu C} \nabla r^{\varepsilon_{0}} \cdot n_{\Omega_{B}^{\varepsilon_{0}}}=0 \quad \text { on } \partial \Omega_{B}^{\varepsilon_{0}} \times J .
$$

The fact that these problems determine $\theta^{\varepsilon_{0}}$ and $\widehat{\sigma}^{\varepsilon_{0}}$ (and therefore $r^{\varepsilon_{0}}$ ) uniquely is a special case of Theorem 7.2, which is proved in Section 7.

Our results so far are summarized in the following theorem.

THEOREM 5.4. As $\varepsilon_{1} \rightarrow 0$, the following weak limits hold in the indicated spaces:

$$
\begin{gathered}
\left(\theta^{\varepsilon_{0} \varepsilon_{1}}\right)^{\sim(2)} \rightarrow \theta^{\varepsilon_{0}} \quad \text { in } L^{2}\left(\Omega ; L^{2}\left(Y_{0 B} ; H^{1}\left(Y_{1 B} \times J\right)\right)\right), \\
\chi_{\Omega_{B, F}^{\varepsilon_{0} \varepsilon_{1}} \sigma^{\varepsilon_{0} \varepsilon_{1}}} \rightarrow \widehat{\sigma}^{\varepsilon_{0}} \quad \text { in } H^{1}\left(J ; L^{2}\left(\Omega_{B}^{\varepsilon_{0}}\right)\right), \\
\chi_{\Omega_{B, F}^{\varepsilon_{B} \varepsilon_{1}}} \frac{K_{\sigma}^{\varepsilon_{0}}}{\mu c} \nabla \sigma^{\varepsilon_{0}} \rightarrow \frac{\left|Y_{1}\right|}{\left|Y_{1 F}\right|} \frac{\mathbf{K}_{\sigma}^{h \varepsilon_{0}}}{\mu c} \nabla \widehat{\sigma}^{\varepsilon_{0}} \quad \text { in } L^{2}\left(\Omega_{B}^{\varepsilon_{0}} \times J\right),
\end{gathered}
$$

and if

$$
\begin{gathered}
r^{\varepsilon_{0}}=\frac{\left|Y_{1}\right|}{\left|Y_{1 F}\right|} \widehat{\sigma}^{\varepsilon_{0}}, \\
f_{B, B}^{\varepsilon_{0}}=\left(\left|Y_{0}\right|\left|Y_{1}\right|\right)^{-1} \int_{Y_{0} \times Y_{1 B}} \phi_{\theta}\left(x_{2}\right) \theta_{t}^{\varepsilon_{0}}\left(\varepsilon_{0} x_{1}+c^{0, \varepsilon_{0}}\left(x_{0}\right), L_{0}\left(x_{0}\right), x_{2}, t\right) d x_{2} d x_{1}, \\
\phi_{\sigma}^{h \varepsilon_{0}} \equiv \frac{\left|Y_{1 F}\right|}{\left|Y_{1}\right|} \phi_{\sigma}^{\varepsilon_{0}}, \quad\left(\mathbf{K}_{\sigma}^{h \varepsilon_{0}}\right)_{i j} \equiv \mathbf{K}_{\sigma}^{\varepsilon_{0}}\left(\omega_{i j}+\frac{\left|Y_{1 F}\right|}{\left|Y_{1}\right|} \delta_{i j}\right),
\end{gathered}
$$


then $\left(r^{\varepsilon_{0}}, \theta^{\varepsilon_{0}}\right)$ is the unique weak solution to the following coupled initialboundary value problems:

$$
\begin{aligned}
& \phi_{\sigma}^{h \varepsilon_{0}} r_{t}^{\varepsilon_{0}}+\varepsilon_{0}^{2} \nabla \cdot\left(\frac{\mathbf{K}_{\sigma}^{h \varepsilon_{0}}}{\mu c} \nabla r^{\varepsilon_{0}}\right)+f_{B, B}^{\varepsilon_{0}}=0 \quad \text { in } \Omega_{B}^{\varepsilon_{0}} \times J, \\
& \frac{\mathbf{K}_{\sigma}^{h \varepsilon_{0}}}{\mu c} \nabla r^{\varepsilon_{0}} \cdot n_{\Omega_{B}^{\varepsilon_{0}}}=0 \quad \text { on } \partial \Omega_{B}^{\varepsilon_{0}} \times J, \\
& r^{\varepsilon_{0}}=\rho_{\text {init }} \quad \text { in } \Omega_{B}^{\varepsilon_{0}} \times\{0\} ， \\
& \phi_{\theta} \theta_{t}^{\varepsilon_{0}}-\nabla_{x_{2}} \cdot\left(\frac{\mathbf{K}_{\theta}}{\mu c} \nabla_{x_{2}} \theta^{\varepsilon_{0}}\right)=0 \quad \text { in } \Omega \times Y_{0 B} \times Y_{1 B} \times J \text {, } \\
& \theta^{\varepsilon_{0}}=\left(r^{\varepsilon_{0}}\right)^{\sim(1)} \text { on } \Omega \times Y_{0 B} \times \partial Y_{1 B} \times J \text {, } \\
& \theta^{\varepsilon_{0}}=\left(\rho_{\text {init }}\right)^{\sim(1)} \text { in } \Omega \times Y_{0 B} \times Y_{1 B} \times\{0\} \text {. }
\end{aligned}
$$

\section{Homogenization as $\varepsilon_{0} \rightarrow 0$}

With an external source term $S$ defined on $\Omega \times J$, we now create a completely new problem on all of $\Omega$. In order to do this, we use the partial differential equations for the system (5.61), (5.62), (5.63), (5.64), (5.65), and (5.66), but we change the boundary condition (5.62) in order to conserve mass flux and pressure on $\partial \Omega_{B}^{\varepsilon_{0}}$. Thus, we have new unknowns now, namely, $\rho^{\varepsilon_{0}}, \sigma^{h\left(\varepsilon_{0}\right)}$, and $\theta^{h\left(\varepsilon_{0}\right)}$. We pose the following problem based on the previous homogenization:

$$
\begin{gathered}
\phi_{\rho} \rho_{t}^{\varepsilon_{0}}-\nabla \cdot\left(\frac{K_{\rho}}{\mu c} \nabla \rho^{\varepsilon_{0}}\right)=S \quad \text { in } \Omega_{F}^{\varepsilon_{0}} \times J, \\
\frac{K_{\rho}}{\mu c} \nabla \rho^{\varepsilon_{0}} \cdot n_{\Omega}=0 \quad \text { on } \partial \Omega \times J, \\
\frac{K_{\rho}}{\mu c} \nabla \rho^{\varepsilon_{0}} \cdot n_{\Omega_{B}^{\varepsilon_{0}}}=\varepsilon_{0}^{2} \frac{\mathbf{K}_{\sigma}^{h \varepsilon_{0}}}{\mu c} \nabla \sigma^{h\left(\varepsilon_{0}\right)} \cdot n_{\Omega_{B}^{\varepsilon_{0}}} \quad \text { on } \partial \Omega_{B}^{\varepsilon_{0}} \times J, \\
\rho^{\varepsilon_{0}}=\rho_{\text {init }} \text { in } \Omega_{F}^{\varepsilon_{0}} \times\{0\}, \\
\phi_{\sigma}^{h \varepsilon_{0}} \sigma_{t}^{h\left(\varepsilon_{0}\right)}-\varepsilon_{0}^{2} \nabla \cdot\left(\frac{\mathbf{K}_{\sigma}^{h \varepsilon_{0}}}{\mu c} \nabla \sigma^{h\left(\varepsilon_{0}\right)}\right)+f_{B, B}^{h \varepsilon_{0}}=0 \quad \text { in } \Omega_{B}^{\varepsilon_{0}} \times J, \\
\sigma^{h\left(\varepsilon_{0}\right)}=\rho^{\varepsilon_{0}} \quad \text { on } \partial \Omega_{B}^{\varepsilon_{0}} \times J, \\
\sigma^{h\left(\varepsilon_{0}\right)}=\rho_{\text {init }} \text { in } \Omega_{B}^{\varepsilon_{0}} \times\{0\}, \\
\left(\frac{\mathbf{K}_{\theta}}{\mu c} \nabla_{x_{2}} \theta^{h\left(\varepsilon_{0}\right)}\right)=0 \quad \text { in } \Omega \times Y_{0 B} \times Y_{1 B} \times J,
\end{gathered}
$$




$$
\begin{aligned}
& \theta^{h\left(\varepsilon_{0}\right)}=\left(\sigma^{h\left(\varepsilon_{0}\right)}\right)^{\sim(1)} \quad \text { on } \Omega \times Y_{0 B} \times \partial Y_{1 B} \times J, \\
& \theta^{h\left(\varepsilon_{0}\right)}=\left(\rho_{\text {init }}\right)^{\sim(1)} \text { in } \Omega \times Y_{0 B} \times Y_{1 B} \times\{0\},
\end{aligned}
$$

where

$$
\begin{aligned}
f_{B, B}^{h \varepsilon_{0}}=\left(\left|Y_{0}\right|\left|Y_{1}\right|\right)^{-1} \int_{Y_{0} \times Y_{1 B}} & \phi_{\theta}\left(x_{2}\right) \\
& \times \theta_{t}^{h \varepsilon_{0}}\left(\varepsilon_{0} x_{1}+c^{0, \varepsilon_{0}}\left(x_{0}\right), L_{0}\left(x_{0}\right), x_{2}, t\right) d x_{2} d x_{1} .
\end{aligned}
$$

Theorem 6.1. Problem (6.1), (6.2), (6.3), (6.4), (6.5), (6.6), (6.7), (6.8), (6.9), and (6.10) is well posed in

$$
\begin{gathered}
L^{\infty}\left(J ; H^{1}\left(\Omega_{F}^{\varepsilon_{0}}\right)\right) \cap L^{2}\left(\Omega_{F}^{\varepsilon_{0}} ; H^{1}(J)\right) \times L^{\infty}\left(J ; H^{1}\left(\Omega_{B}^{\varepsilon_{0}}\right)\right) \cap L^{2}\left(\Omega_{B}^{\varepsilon_{0}} ; H^{1}(J)\right) \\
\times L^{\infty}\left(J ; L^{2}\left(\Omega \times Y_{0 B} ; H^{1}\left(Y_{1 B}\right)\right)\right) \cap L^{2}\left(\Omega \times Y_{0 B} \times Y_{1 B} ; H^{1}(J)\right) .
\end{gathered}
$$

This is a specific case of Theorem 7.1 in the more general setting of Section 7. We prove it there, and we also deduce from it the following important estimates. There exists $C>0$ that is independent of $\varepsilon_{0}$ such that

$$
\begin{gathered}
\left\|\rho^{\varepsilon_{0}}\right\|_{L^{\infty}\left(J ; H^{1}\left(\Omega_{F}^{\varepsilon_{0}}\right)\right)}+\left\|\rho_{t}^{\varepsilon_{0}}\right\|_{L^{2}\left(\Omega_{F}^{\varepsilon_{0}} \times J\right)} \leq C\left(\|S\|_{L^{2}(\Omega \times J)}+\left\|\rho_{\text {init }}\right\|_{H^{1}(\Omega)}\right) \\
\left\|\sigma^{h\left(\varepsilon_{0}\right)}\right\|_{L^{2}\left(\Omega_{B}^{\varepsilon_{0}} \times J\right)}+\left\|\sigma_{t}^{h\left(\varepsilon_{0}\right)}\right\|_{L^{2}\left(\Omega_{B}^{\varepsilon_{0}} \times J\right)} \leq C\left\|\rho_{\text {init }}\right\|_{H^{1}(\Omega)^{\prime}} \\
\varepsilon_{0}\left\|\nabla \sigma^{h\left(\varepsilon_{0}\right)}\right\|_{L^{\infty}\left(J ; L^{2}\left(\Omega_{B}^{\left.\left.\varepsilon_{0} \times J\right)\right)}\right.\right.} \leq C\left\|\rho_{\text {init }}\right\|_{H^{1}(\Omega)^{\prime}} \\
\left\|\theta^{h\left(\varepsilon_{0}\right)}\right\|_{L^{\infty}\left(J ; L^{2}\left(\Omega \times Y_{0 B}\right) ; H^{1}\left(Y_{1 B}\right)\right)}+\left\|\theta_{t}^{h\left(\varepsilon_{0}\right)}\right\|_{L^{2}\left(\Omega \times Y_{0 B} \times Y_{1 B} \times J\right)} \leq C\left\|\rho_{\text {init }}\right\|_{H^{1}(\Omega)}{ }^{\prime}
\end{gathered}
$$

We now proceed to determine the limit of $\left(\rho^{\varepsilon_{0}}, \sigma^{h\left(\varepsilon_{0}\right)}, \theta^{h\left(\varepsilon_{0}\right)}\right)$ as $\varepsilon_{0} \rightarrow 0$. By virtue of (6.13), (6.14), (6.15), and (6.16), we can pass to a subsequence and deduce that as $\varepsilon_{0} \rightarrow 0$, we have the following weak limits in the indicated spaces:

$$
\begin{gathered}
\chi_{\Omega_{F}^{\varepsilon_{0}}} \phi_{\rho} \rho^{\varepsilon_{0}}-\frac{\left|Y_{0 F}\right|}{\left|Y_{0}\right|} \phi_{\rho} \rho \quad \text { in } H^{1}\left(J ; L^{2}(\Omega)\right), \\
\chi_{\Omega_{F}^{\varepsilon_{0}}} \frac{K_{\rho}}{\mu C} \nabla \rho^{\varepsilon_{0}}-\zeta \quad \text { in } L^{2}(\Omega \times J), \\
\left(\sigma^{h\left(\varepsilon_{0}\right)}\right)^{\sim(1)} \rightarrow \sigma \quad \text { in } L^{2}\left(\Omega ; H^{1}\left(Y_{0 B} \times J\right)\right), \\
\theta^{h\left(\varepsilon_{0}\right)} \rightarrow \theta \text { in } L^{2}\left(\Omega \times Y_{0 B} ; H^{1}\left(Y_{1 B} \times J\right)\right) .
\end{gathered}
$$


The partial differential equations satisfied by $(\rho, \sigma, \theta)$ will be derived next. To obtain the equation for $\theta$, we pass to the limit in the equation defining the weak form of (6.7) by virtue of (6.20) to obtain

$$
\begin{gathered}
\left(\phi_{\theta} \theta_{t}, \psi\right)_{\Omega \times Y_{0 B} \times Y_{1 B} \times J}+\left(\frac{\mathbf{K}_{\theta}}{\mu c} \nabla_{x_{2}} \theta, \psi\right)_{\Omega \times Y_{0 B} \times Y_{1 B} \times J} \\
=0 \quad \forall \psi \in L^{2}\left(\Omega \times Y_{0 B} \times J ; H_{0}^{1}\left(Y_{1 B}\right)\right) .
\end{gathered}
$$

To find the equation for $\sigma$, we deduce from the weak form of (6.5) and the argument in [5, page 831$]$ that

$$
\begin{aligned}
\left(\phi_{\sigma}^{h}\left(\sigma_{t}^{h\left(\varepsilon_{0}\right)}\right)^{\sim(1)}, \psi\right)_{\Omega \times Y_{0 B} \times J}+\left(\frac{\mathbf{K}_{\sigma}^{h}}{\mu c} \nabla_{x_{1}}\left(\sigma_{t}^{h\left(\varepsilon_{0}\right)}\right)^{\sim(1)}, \nabla_{x_{1}} \psi\right)_{\Omega \times Y_{0 B} \times J} \\
\quad+\left(\left(f_{B, B}^{h \varepsilon_{0}}\right)^{\sim(1)}, \psi\right)_{\Omega \times Y_{0 B} \times J} \\
=T_{9}+T_{10}+T_{11}=0 \quad \forall \psi \in L^{2}\left(\Omega ; L^{2}\left(J ; H_{0}^{1}\left(Y_{0 B}\right)\right)\right) .
\end{aligned}
$$

Let $\varepsilon_{0} \rightarrow 0$; from (6.19),

$$
T_{9} \longrightarrow\left(\phi_{\sigma}^{h} \sigma_{t}, \psi\right)_{\Omega \times Y_{0 B} \times J^{\prime}}, \quad T_{10} \longrightarrow\left(\frac{\mathbf{K}_{\sigma}^{h}}{\mu c} \nabla_{x_{1}} \sigma, \nabla_{x_{1}} \psi\right)_{\Omega \times Y_{0 B} \times J} .
$$

For term $T_{11}$, we write

$$
\begin{aligned}
\left(f_{B, B}^{h \varepsilon_{0}}\right)^{\sim(1)}\left(x_{0}, y, t\right) & \\
& =f_{B, B}^{h \varepsilon_{0}}\left(\varepsilon_{0} y+c^{0, \varepsilon_{0}}\left(x_{0}\right), t\right) \\
& =\frac{1}{\left|Y_{0}\right|\left|Y_{1}\right|} \int_{Y_{0} \times Y_{1 B}} \phi_{\theta}\left(x_{2}\right) \theta_{t}^{h\left(\varepsilon_{0}\right)}\left(\varepsilon_{0} x_{1}+c^{0, \varepsilon_{0}}\left(x_{0}\right), y, x_{2}, t\right) d x_{2} d x_{1},
\end{aligned}
$$

where we have used the fact that $c^{0, \varepsilon_{0}}\left(\varepsilon_{0} x_{1}+c^{0, \varepsilon_{0}}\left(x_{0}\right)\right)=c^{0, \varepsilon_{0}}\left(x_{0}\right)$. Hence,

$$
\begin{array}{r}
T_{11}=\frac{1}{\left|Y_{0}\right|\left|Y_{1}\right|} \int_{J \times \Omega \times Y_{0 B}}\left(\int_{Y_{0} \times Y_{1 B}} \phi_{\theta}\left(x_{2}\right) \theta_{t}^{h\left(\varepsilon_{0}\right)}\left(\varepsilon_{0} x_{1}+c^{0, \varepsilon_{0}}\left(x_{0}\right), y, x_{2}, t\right)\right. \\
\left.\times \psi\left(x_{0}, y, t\right) d x_{2} d x_{1}\right) d y d x_{0} d t \\
=\frac{1}{\left|Y_{0}\right|\left|Y_{1}\right|} \int_{J \times Y_{0 B}}\left[\int _ { Y _ { 1 B } } \phi _ { \theta } ( x _ { 2 } ) \left(\int_{\Omega \times Y_{0}} \theta_{t}^{h\left(\varepsilon_{0}\right)}\left(\varepsilon_{0} x_{1}+c^{0, \varepsilon_{0}}\left(x_{0}\right), y, x_{2}, t\right)\right.\right. \\
\left.\left.\times \psi\left(x_{0}, y, t\right) d x_{1} d x_{0}\right) d x_{2}\right] d y d t .
\end{array}
$$


Hence, by Lemmas 2.1, 2.2 and (6.20),

$$
\begin{aligned}
T_{11} & =\frac{1}{\left|Y_{0}\right|\left|Y_{1}\right|} \int_{J \times Y_{0} \times \Omega \times Y_{0 B} \times Y_{1 B}} \phi_{\theta}\left(x_{2}\right) \theta_{t}^{h\left(\varepsilon_{0}\right)}\left(x_{0}, y, x_{2}, t\right) \\
& \stackrel{\varepsilon_{0} \rightarrow 0}{\longrightarrow} \frac{1}{\left|Y_{0}\right|\left|Y_{1}\right|} \int_{J \times Y_{0} \times \Omega \times Y_{0 B} \times Y_{1 B}} \phi_{\theta}\left(\varepsilon_{0} x_{1}+c^{0, \varepsilon_{0}}\left(x_{0}\right), y, t\right) d x_{2} d y d x_{0} d x_{1} d t \\
& =\frac{\left|Y_{0}\right|}{\left|Y_{0}\right|\left|Y_{1}\right|} \int_{J \times \Omega \times Y_{0 B} \times Y_{1 B}} \phi_{\theta}\left(x_{2}\right) \theta_{t}\left(x_{0}, x_{1}, x_{2}, t\right) d x_{2} d y d x_{0} d x_{1} d t \\
& =\frac{1}{\left|Y_{1}\right|}\left(\phi_{\theta} \theta_{t}, \psi\right)_{J \times \Omega \times Y_{0 B} \times Y_{1 B}} .
\end{aligned}
$$

Hence, by (6.22), (6.23), and (6.26),

$$
\begin{aligned}
& \left(\phi_{\sigma}^{h} \sigma_{t}, \psi\right)_{\Omega \times Y_{0 B} \times J}+\left(\frac{\mathbf{K}_{\sigma}^{h}}{\mu c} \nabla_{x_{1}} \sigma, \nabla_{x_{1}} \psi\right)_{\Omega \times Y_{0 B} \times J} \\
& \quad+\frac{1}{\left|Y_{1}\right|}\left(\phi_{\theta} \theta_{t}, \psi\right)_{J \times \Omega \times Y_{0 B} \times Y_{1 B}}=0 \quad \forall \psi \in L^{2}\left(\Omega ; L^{2}\left(J ; H_{0}^{1}\left(Y_{0 B}\right)\right)\right) .
\end{aligned}
$$

In order to derive an equation for $\rho$, we use the weak form of (6.1), (6.2), and (6.3) to write

$$
\begin{aligned}
T_{12}+ & T_{13}+T_{14}+T_{15}+T_{16} \\
= & \left(\phi_{\rho} \rho_{t}^{\varepsilon_{0}}, \varphi\right)_{\Omega_{F}^{\varepsilon_{0}} \times J}+\left(\phi_{\sigma}^{h \varepsilon_{0}} \sigma_{t}^{h\left(\varepsilon_{0}\right)}, \varphi\right)_{\Omega_{B}^{\varepsilon_{0} \times J}}+\left(\frac{K_{\rho}}{\mu c} \nabla \rho^{\varepsilon_{0}}, \nabla \varphi\right)_{\Omega_{F}^{\varepsilon_{0} \times J}} \\
& +\varepsilon_{0}^{2}\left(\frac{K_{\sigma}^{h \varepsilon_{0}}}{\mu c} \nabla \sigma^{h\left(\varepsilon_{0}\right)}, \nabla \varphi\right)_{\Omega_{B}^{\varepsilon_{0} \times J}}+\left(f_{B, B}^{h \varepsilon_{0}}, \varphi\right)_{\Omega_{B}^{\varepsilon_{0}} \times J} \\
= & (S, \varphi)_{\Omega_{F}^{\varepsilon_{0}} \times J}=T_{17} \quad \forall \varphi \in L^{2}\left(J ; H^{1}(\Omega)\right) .
\end{aligned}
$$

As $\varepsilon_{0} \rightarrow 0$, the following convergence results take place by (6.17) for $T_{12}$; Lemma 2.1, (6.19), and Lemma 2.2 for $T_{13} ;(6.18)$ for $T_{14} ;(6.15)$ for $T_{15}$; 
Lemma 2.4 and (6.20) for $T_{16}$; and Lemmas 2.1, 2.2 for $T_{17}$ :

$$
\begin{aligned}
T_{12} \longrightarrow & \left(\frac{\left|Y_{0 F}\right|}{\left|Y_{0}\right|} \phi_{\rho} \rho_{t}, \varphi\right)_{\Omega \times J}, \\
T_{13}= & \left|Y_{0}\right|^{-1}\left(\phi_{\sigma}^{h}\left(\sigma_{t}^{h\left(\varepsilon_{0}\right)}\right)^{\sim(1)}, \varphi^{\sim(1)}\right)_{\Omega \times Y_{0 B} \times J} \longrightarrow\left|Y_{0}\right|^{-1}\left(\phi_{\sigma}^{h} \sigma_{t}, \varphi\right)_{\Omega \times Y_{0 B} \times J^{\prime}} \\
T_{14} \longrightarrow & (\xi, \nabla \varphi)_{\Omega \times J}, T_{15} \longrightarrow 0, \\
T_{16}= & \frac{1}{\left|Y_{0}\right|\left|Y_{1}\right|} \int_{J \times \Omega_{B}^{\varepsilon_{0}} \times Y_{0} \times Y_{1 B}} \phi_{\theta}\left(x_{2}\right) \theta_{t}^{h\left(\varepsilon_{0}\right)}\left(\varepsilon_{0} x_{1}+c^{0, \varepsilon_{0}}\left(x_{0}\right), L_{0}\left(x_{0}\right), x_{2}, t\right) \\
& \times \varphi\left(x_{0}, t\right) d x_{2} d x_{1} d x_{0} d t \\
= & \frac{1}{\left|Y_{0}\right|\left|Y_{1}\right|} \int_{J \times Y_{1 B}}\left(\int_{\Omega \times Y_{0 B}} \phi_{\theta}\left(x_{2}\right) \theta_{t}^{h\left(\varepsilon_{0}\right)}\left(x_{0}, x_{1}, x_{2}, t\right)\right. \\
& \left.\times \varphi^{\sim(1)}\left(x_{0}, x_{1}, t\right) d x_{1} d x_{0}\right) d x_{2} d t \\
= & \frac{1}{\left|Y_{0}\right|\left|Y_{1}\right|}\left(\phi_{\theta} \theta_{t}^{h\left(\varepsilon_{0}\right)}, \varphi^{\sim(1)}\right)_{J \times \Omega \times Y_{0 B} \times Y_{1 B}} \longrightarrow \frac{1}{\left|Y_{0}\right|\left|Y_{1}\right|}\left(\phi_{\theta} \theta_{t}, \varphi\right)_{J \times \Omega \times Y_{0 B} \times Y_{1 B}{ }^{\prime}} \\
T_{17}= & \frac{1}{\left|Y_{0}\right|}\left(S^{\sim(1)}, \varphi^{\sim(1)}\right)_{\Omega \times Y_{0 F} \times J} \longrightarrow \frac{1}{\left|Y_{0}\right|}(S, \varphi)_{\Omega \times Y_{0 F} \times J} \\
= & \frac{\left|Y_{0 F}\right|}{\left|Y_{0}\right|}(S, \varphi)_{\Omega \times J} .
\end{aligned}
$$

We now apply the arguments of [5, pages 831-833] to identify $\xi$. We need only to show that the interchange term $f_{B, B}^{h \varepsilon_{0}}$ between the microand mesoscales is bounded for $\varepsilon_{0}>0$. To this end, we estimate it using the Cauchy-Schwartz inequality, Lemma 2.4, and (6.20) as follows:

$$
\begin{aligned}
& \left\|f_{B, B}^{h \varepsilon_{0}}\right\|_{\Omega_{B}^{\varepsilon_{0} \times J}}^{2} \\
& \quad \leq C \int_{J \times \Omega_{B}^{\varepsilon_{0} \times Y_{0} \times Y_{1 B}}}\left|\theta_{t}^{h\left(\varepsilon_{0}\right)}\left(\varepsilon_{0} x_{1}+c^{0, \varepsilon_{0}}\left(x_{0}\right), L_{0}\left(x_{0}\right), x_{2}, t\right)\right|^{2} d x_{2} d x_{1} d x_{0} d t \\
& \quad=C \int_{J \times \Omega \times Y_{0 B} \times Y_{1 B}} 1^{\sim(1)}\left|\theta_{t}^{h\left(\varepsilon_{0}\right)}\right| d x_{2} d x_{1} d x_{0} d t \\
& \quad=C\left\|\theta_{t}^{h\left(\varepsilon_{0}\right)}\right\|_{L^{2}\left(\Omega \times Y_{0 B} \times Y_{1 B} \times J\right)}^{2} \leq C .
\end{aligned}
$$

It follows that

$$
(\xi, \nabla \varphi)_{\Omega \times J}=\left(\frac{\mathbf{K}_{\rho}^{h}}{\mu c} \nabla \rho, \nabla \varphi\right)_{\Omega \times J},
$$


where $\mathbf{K}_{\rho}^{h}$ is the homogenized permeability tensor corresponding to $K_{\rho}$, as defined in [5, page 827] with the $Q$ appearing there replaced by $Y_{0}$.

Hence, by (6.28), (6.29), and (6.31),

$$
\begin{aligned}
& \frac{\left|Y_{0 F}\right|}{\left|Y_{0}\right|}\left(\phi_{\rho} \rho_{t}, \varphi\right)_{\Omega \times J}+\frac{1}{\left|Y_{0}\right|}\left(\phi_{\sigma}^{h} \sigma_{t}, \varphi\right)_{\Omega \times Y_{0 B} \times J}+\left(\frac{\mathbf{K}_{\rho}^{h}}{\mu c} \nabla \rho, \nabla \varphi\right)_{\Omega \times J} \\
& \quad+\frac{1}{\left|Y_{0}\right|\left|Y_{1}\right|}\left(\phi_{\theta} \theta_{t}, \varphi\right)_{\Omega \times Y_{0 B} \times Y_{1 B} \times J} \\
& =\frac{\left|Y_{0 F}\right|}{\left|Y_{0}\right|}(S, \varphi)_{\Omega \times J} \quad \forall \varphi \in L^{2}\left(J ; H^{1}(\Omega)\right) .
\end{aligned}
$$

We now determine the initial and boundary conditions satisfied by $(\rho, \sigma, \theta)$. By weak continuity of the appropriate trace operators and Lemma 2.2, we can pass to the limit in (6.9), (6.10) as in Section 5 to get

$$
\theta=\sigma \quad \text { on } \Omega \times Y_{0 B} \times \partial Y_{1 B} \times J, \quad \theta=\rho_{\text {init }} \quad \text { in } \Omega \times Y_{0 B} \times Y_{1 B} \times\{0\},
$$

and from (6.4), (6.6), (6.8), and the argument on [5, page 833], it follows that

$$
\begin{gathered}
\rho=\sigma \quad \text { on } \Omega \times \partial Y_{0 B} \times J, \quad \rho=\rho_{\text {init }} \quad \text { in } \Omega \times\{0\}, \\
\sigma=\rho_{\text {init }} \quad \text { in } \Omega \times Y_{0 B} \times\{0\} .
\end{gathered}
$$

We have shown that $(\rho, \sigma, \theta)$ satisfies [16, problem (3.29)-(3.31)]. Hence, by [16, Theorem 5.2], $(\rho, \sigma, \theta)$ is uniquely determined by (6.21), (6.27), (6.32), (6.33), and (6.34). Hence, the limits in (6.17), (6.18), (6.19), and (6.20) hold as $\varepsilon_{0} \rightarrow 0$ through its full range of values.

The results of this section are summarized in the following theorem.

THEOREM 6.2. As $\varepsilon_{0} \rightarrow 0$, the following limits hold weakly in the indicated spaces:

$$
\begin{gathered}
\chi_{\Omega_{F}^{\varepsilon_{0}}} \phi_{\rho} \rho^{\varepsilon_{0}} \rightarrow \frac{\left|Y_{0 F}\right|}{\left|Y_{0}\right|} \phi_{\rho} \rho \text { in } H^{1}\left(J ; L^{2}(\Omega)\right), \\
\chi_{\Omega_{F}^{\varepsilon_{0}}} \frac{K_{\rho}}{\mu c} \nabla \rho^{\varepsilon_{0}} \rightarrow \frac{\mathbf{K}_{\rho}^{h}}{\mu c} \nabla \rho \text { in } L^{2}(\Omega \times J), \\
\left(\sigma^{h\left(\varepsilon_{0}\right)}\right)^{\sim(1)} \rightarrow \sigma \quad \text { in } L^{2}\left(\Omega ; H^{1}\left(Y_{0 B} \times J\right)\right), \\
\theta^{h\left(\varepsilon_{0}\right)} \rightarrow \theta \text { in } L^{2}\left(\Omega \times Y_{0 B} ; H^{1}\left(Y_{1 B} \times J\right)\right),
\end{gathered}
$$


and if

$$
\begin{gathered}
\phi_{\rho}^{h}=\frac{\left|Y_{0 F}\right|}{\left|Y_{0}\right|} \phi_{\rho}, \quad \phi_{\sigma}^{h}=\frac{\left|Y_{1 F}\right|}{\left|Y_{1}\right|} \phi_{\sigma} \\
\left(\mathbf{K}_{\sigma}^{h}\right)_{i j} \equiv K_{\sigma}\left(\omega_{i j}+\frac{\left|Y_{1 F}\right|}{\left|Y_{1}\right|} \delta_{i j}\right), \quad S^{h}=\frac{\left|Y_{0 F}\right|}{\left|Y_{0}\right|} S,
\end{gathered}
$$

then $(\rho, \sigma, \theta)$ is the unique solution to the following system of coupled initialboundary value problems: $\rho$ solves

$$
\begin{gathered}
\phi_{\rho}^{h} \rho_{t}-\nabla_{x_{0}} \cdot\left(\frac{\mathbf{K}_{\rho}^{h}}{\mu c} \nabla_{x_{0}} \rho\right)+\frac{1}{\left|Y_{0}\right|} \int_{Y_{0 B}} \phi_{\sigma}^{h} \sigma_{t} d x_{1} \\
\quad+\frac{1}{\left|Y_{0}\right|\left|Y_{1}\right|} \int_{Y_{0 B} \times Y_{1 B}} \phi_{\theta} \theta_{t} d x_{2} d x_{1}=S^{h} \quad \text { on } \Omega \times J, \\
\frac{\mathbf{K}_{\rho}^{h}}{\mu c} \nabla_{x_{0}} \rho \cdot n_{\Omega}=0 \quad \text { on } \partial \Omega \times J, \quad \rho=\rho_{\text {init }} \quad \text { on } \Omega \times\{0\},
\end{gathered}
$$

where to each $x_{0} \in \Omega$ a block $Y_{0 B}$ is associated such that $\sigma$ solves

$$
\begin{gathered}
\phi_{\sigma}^{h} \sigma_{t}-\nabla_{x_{1}} \cdot\left(\frac{\mathbf{K}_{\sigma}^{h}}{\mu c} \nabla_{x_{1}} \sigma\right)+\frac{1}{\left|Y_{1}\right|} \int_{Y_{1 B}} \phi_{\theta} \theta_{t} d x_{2}=0 \quad \text { in } \Omega \times Y_{0 B} \times J, \\
\sigma=\rho \quad \text { on } \Omega \times \partial Y_{0 B} \times J, \quad \sigma=\rho_{\text {init }} \quad \text { in } \Omega \times Y_{0 B} \times\{0\},
\end{gathered}
$$

where to each $x_{0} \in \Omega$ a block $Y_{0 B}$ is associated, and to each $x_{1} \in Y_{0 B}$ a block $Y_{1 B}$ is associated such that $\theta$ solves

$$
\begin{gathered}
\phi_{\theta} \theta_{t}-\nabla_{x_{2}} \cdot\left(\frac{\mathbf{K}_{\theta}}{\mu c} \nabla_{x_{2}} \theta\right)=0 \quad \text { in } \Omega \times Y_{0 B} \times Y_{1 B} \times J, \\
\theta=\sigma \quad \text { on } \Omega \times Y_{0 B} \times \partial Y_{1 B} \times J, \quad \theta=\rho_{\text {init }} \quad \text { in } \Omega \times Y_{0 B} \times Y_{1 B} \times\{0\} .
\end{gathered}
$$

\section{7. $(N+1)$-scale porosity model}

In this section, we generalize the triple-porosity model to an $(N+1)$ scale porosity model. For the recursive homogenization procedure, we 
hold $\varepsilon_{0}, \ldots, \varepsilon_{m-1}$ constant and let $\varepsilon_{m} \rightarrow 0$, recursively starting with $m=$ $N-1$ and ending with $m=0$. Generalizing the techniques used to derive the triple-porosity model, we recursively derive a flow model for $N>2$ levels of fractures. We begin by assuming that $\varepsilon_{0}, \ldots, \varepsilon_{i-1}$ are held constant and $\varepsilon_{i}$ is sent to zero, for a fixed $i \geq 1$, giving the following homogenized system of coupled partial differential equations (where, for $i=1$, we set $\left.\Omega_{B, H}^{\varepsilon_{0}} \equiv \Omega_{H^{\prime}}^{\varepsilon_{0}} H=B, F\right)$ :

$$
\begin{gathered}
\phi_{i}^{h\left(\varepsilon_{0} \cdots \varepsilon_{i-1}\right)} \frac{\partial \rho_{i}^{h\left(\varepsilon_{0} \cdots \varepsilon_{i-1}\right)}}{\partial t}-\varepsilon_{0}^{2} \cdots \varepsilon_{i-1}^{2} \nabla_{x_{0}} \cdot\left(\frac{\mathbf{K}_{i}^{h\left(\varepsilon_{0} \cdots \varepsilon_{i-1}\right)}}{\mu c} \nabla_{x_{0}} \rho_{i}^{h\left(\varepsilon_{0} \cdots \varepsilon_{i-1}\right)}\right) \\
+L_{i}^{h\left(\varepsilon_{0} \cdots \varepsilon_{i-1}\right)}=0 \quad \text { in } \Omega_{B, B}^{\varepsilon_{0} \cdots \varepsilon_{i-1}} \times J, \\
\frac{\mathbf{K}_{i}^{h\left(\varepsilon_{0} \cdots \varepsilon_{i-1}\right)}}{\mu c} \nabla_{x_{0}} \rho_{i}^{h\left(\varepsilon_{0} \cdots \varepsilon_{i-1}\right)} \cdot n_{\Omega_{B, B}^{\varepsilon_{0} \cdots \varepsilon_{i-1}}=0 \quad \text { in } \partial \Omega_{B, B}^{\varepsilon_{0} \cdots \varepsilon_{i-1}} \times J,} \rho_{i}^{h\left(\varepsilon_{0} \cdots \varepsilon_{i-1}\right)}=\rho_{\text {init }} \quad \text { in } \Omega_{B, B}^{\varepsilon_{0} \cdots \varepsilon_{i-1}} \times\{0\},
\end{gathered}
$$

where

$$
\begin{aligned}
& L_{i}^{h\left(\varepsilon_{0} \cdots \varepsilon_{i-1}\right)} \\
& =\sum_{\alpha=i}^{N-1} \frac{1}{\left|Y_{0}\right| \cdots\left|Y_{\alpha}\right|} \\
& \quad \times \int_{Y_{0} \times \cdots \times Y_{i-1} \times Y_{i B} \times \cdots \times Y_{\alpha B}} \phi_{\alpha+1}^{h}\left(y_{\alpha+1}\right) \frac{\partial \rho_{\alpha+1}^{h\left(\varepsilon_{0} \cdots \varepsilon_{i-1}\right)}}{\partial t} \\
& \times\left(\sum_{k=0}^{i-1}\left(\varepsilon_{k} y_{k+1}+c^{k, \varepsilon_{k}}\left(L_{k-1}\left(x_{0}\right)\right)\right) \mathbf{e}_{k}\right. \\
& \left.\quad+L_{i-1}\left(x_{0}\right) \mathbf{e}_{i}+\sum_{k=i+1}^{\alpha+1} y_{k} \mathbf{e}_{k}+t \mathbf{e}_{\alpha+2}\right) d y_{\alpha+1} \cdots d y_{1} .
\end{aligned}
$$

We have the following system of coupled initial-boundary value problems that are coupled with the above initial-boundary value problem. We state each of them in terms of $j$, where $j=i+1, \ldots, N$, as follows. For every $x_{0} \in \Omega$, there exists a block $Y_{0 B}$, and for every $x_{1} \in Y_{0 B}$, there exists a block $Y_{2 B}$, and so forth, and for every $x_{j} \in \Upsilon_{(j-1) B}$, the following initial-boundary value problem is satisfied:

$$
\begin{aligned}
\phi_{j}^{h} \frac{\partial \rho_{j}^{h\left(\varepsilon_{0} \cdots \varepsilon_{i-1}\right)}}{\partial t}-\nabla_{x_{j}} \cdot\left(\frac{\mathbf{K}_{j}^{h}}{\mu c} \nabla_{x_{j}} \rho_{j}^{h\left(\varepsilon_{0} \cdots \varepsilon_{i-1}\right)}\right) \\
+S_{j}^{h\left(\varepsilon_{0} \cdots \varepsilon_{i-1}\right)}=0 \quad \text { in } \Omega \times \Upsilon_{0 B} \times \cdots \times Y_{(j-1) B} \times J,
\end{aligned}
$$




$$
\begin{aligned}
& \rho_{j}^{h\left(\varepsilon_{0} \cdots \varepsilon_{i-1}\right)}= \begin{cases}\left(\rho_{j-1}^{h\left(\varepsilon_{0} \cdots \varepsilon_{i-1}\right)}\right)^{\sim(i)} & \text { if } j=i+1, \\
\rho_{j-1}^{h\left(\varepsilon_{0} \cdots \varepsilon_{i-1}\right)} & \text { otherwise, }\end{cases} \\
& \text { in } \Omega \times Y_{0 B} \times \cdots \times Y_{(j-2) B} \times \partial Y_{(j-1) B} \times J \text {, } \\
& \rho_{j}^{h\left(\varepsilon_{0} \cdots \varepsilon_{i-1}\right)}=\left(\rho_{\text {init }}\right)^{\sim(i)} \text { in } \Omega \times Y_{0 B} \times \cdots \times Y_{(j-1) B} \times\{0\} \text {, }
\end{aligned}
$$

where, for $j=i+1, \ldots, N-1$,

$$
\begin{aligned}
& S_{j}^{h\left(\varepsilon_{0} \cdots \varepsilon_{i-1}\right)} \\
& =\sum_{\alpha=j}^{N-1}\left(\frac{\left|Y_{i}\right|\left|Y_{i+1}\right| \cdots\left|Y_{j-1}\right|}{\left|Y_{0}\right|\left|Y_{1}\right| \cdots\left|Y_{\alpha}\right|}\right) \\
& \times \int_{Y_{0} \times \cdots \times Y_{i-1} \times Y_{j B} \times \cdots \times Y_{\alpha B}} \phi_{\alpha+1}^{h}\left(y_{\alpha+1}\right) \frac{\partial \rho_{\alpha+1}^{h\left(\varepsilon_{0} \cdots \varepsilon_{i-1}\right)}}{\partial t} \\
& \times\left(\sum_{k=0}^{i-1}\left(\varepsilon_{k} y_{k+1}+c^{k, \varepsilon_{k}}\left(x_{k}\right)\right) \mathbf{e}_{k}+\sum_{k=i}^{j} x_{k} \mathbf{e}_{k}\right. \\
& \left.+\sum_{k=j+1}^{\alpha+1} y_{k} \mathbf{e}_{k}+t \mathbf{e}_{\alpha+2}\right) d y_{\alpha+1} \cdots d y_{j+1} d y_{i} \cdots d y_{1},
\end{aligned}
$$

and $S_{N}^{h\left(\varepsilon_{0} \cdots \varepsilon_{i-1}\right)} \equiv 0$. Also, $\phi_{N}^{h} \equiv \phi_{N}$ and $\mathbf{K}_{N}^{h} \equiv \mathbf{K}_{N}$.

If we proceed to recursively homogenize the problem at the next level, that is, if we want to let $\varepsilon_{i-1} \rightarrow 0$, then we first change the no-flow boundary condition on $\partial \Omega_{B, B}^{\varepsilon_{0} \cdots \varepsilon_{i-1}} \times J$ in (7.1) to the following boundary condition:

$$
\rho_{i}^{h\left(\varepsilon_{0} \cdots \varepsilon_{i-1}\right)}=\rho_{i-1}^{\left(\varepsilon_{0} \cdots \varepsilon_{i-1}\right)} \quad \text { in } \partial \Omega_{B, B}^{\varepsilon_{0} \cdots \varepsilon_{i-1}} \times J,
$$

Then, we impose the following initial-boundary value problem in $\Omega_{B, F}^{\varepsilon_{0} \cdots \varepsilon_{i-1}}$ $\times J$ :

$$
\begin{aligned}
& \phi_{i-1}^{\varepsilon_{0} \cdots \varepsilon_{i-2}} \frac{\partial \rho_{i-1}^{\left(\varepsilon_{0} \cdots \varepsilon_{i-1}\right)}}{\partial t}-\varepsilon_{0}^{2} \cdots \varepsilon_{i-2}^{2} \nabla_{x_{0}} \cdot\left(\frac{K_{i-1}^{\varepsilon_{0} \cdots \varepsilon_{i-2}}}{\mu c} \nabla_{x_{0}} \rho_{i-1}^{\left(\varepsilon_{0} \cdots \varepsilon_{i-1}\right)}\right) \\
& =\delta_{i, 1} S\left(x_{0}, t\right) \text { in } \Omega_{B, F}^{\varepsilon_{0} \cdots \varepsilon_{i-1}} \times J,
\end{aligned}
$$


356 Analysis of a multiple-porosity model

$$
\begin{aligned}
& \frac{K_{i-1}^{\varepsilon_{0} \cdots \varepsilon_{i-2}}}{\mu c} \nabla_{x_{0}} \rho_{i-1}^{\left(\varepsilon_{0} \cdots \varepsilon_{i-1}\right)} \cdot n_{\Omega_{B, B}^{\varepsilon_{0} \cdots \varepsilon_{i-1}}} \\
& \quad=\varepsilon_{i-1}^{2} \frac{\mathbf{K}_{i}^{h\left(\varepsilon_{0} \cdots \varepsilon_{i-1}\right)}}{\mu c} \nabla_{x_{0}} \rho_{i}^{h\left(\varepsilon_{0} \cdots \varepsilon_{i-1}\right)} \cdot n_{\Omega_{B, B}^{\varepsilon_{0} \cdots \varepsilon_{i-1}}} \quad \text { on } \partial \Omega_{B, B}^{\varepsilon_{0} \cdots \varepsilon_{i-1}} \times J, \\
& \frac{K_{i-1}^{\varepsilon_{0} \cdots \varepsilon_{i-2}}}{\mu c} \nabla_{x_{0}} \rho_{i-1}^{\left(\varepsilon_{0} \cdots \varepsilon_{i-1}\right)} \cdot n_{\Omega_{B, F}^{\varepsilon_{0} \cdots \varepsilon_{i-1}}}=0 \quad \text { on } \partial \Omega_{B, B}^{\varepsilon_{0} \cdots \varepsilon_{i-2}} \times J, \\
& \rho_{i-1}^{\left(\varepsilon_{0} \cdots \varepsilon_{i-1}\right)}=\rho_{\text {init }} \quad \text { in } \Omega_{B, F}^{\varepsilon_{0} \cdots \varepsilon_{i-1}} \times\{0\} .
\end{aligned}
$$

We now prove that the model is well posed.

THEOREM 7.1. The problem at stage $i-1$, namely, (7.1) (with the new boundary condition (7.5)), (7.3), and (7.6) is well posed in

$$
\begin{aligned}
& L^{\infty}(J ;\left.H^{1}\left(\Omega_{B, F}^{\varepsilon_{0} \cdots \varepsilon_{i-1}}\right)\right) \cap L^{2}\left(\Omega_{B, F}^{\varepsilon_{0} \cdots \varepsilon_{i-1}} ; H^{1}(J)\right) \times L^{\infty}\left(J ; H^{1}\left(\Omega_{B, B}^{\varepsilon_{0} \cdots \varepsilon_{i-1}}\right)\right) \\
& \cap L^{2}\left(\Omega_{B, B}^{\varepsilon_{0} \cdots \varepsilon_{i-1}} ; H^{1}(J)\right) \\
& \times\left[\times_{j=i+1}^{N} L^{\infty}\left(J ; L^{2}\left(\Omega \times Y_{0 B} \times \cdots \times Y_{(j-2) B} ; H^{1}\left(Y_{(j-1) B} \times J\right)\right)\right)\right. \\
&\left.\cap L^{2}\left(\Omega \times Y_{0 B} \times \cdots \times Y_{(j-1) B} ; H^{1}(J)\right)\right],
\end{aligned}
$$

and there exists $C>0$, independent of $\varepsilon_{i-1}$, such that

$$
\begin{gathered}
\left\|\rho_{i-1}^{\left(\varepsilon_{0} \cdots \varepsilon_{i-1}\right)}\right\|_{L^{\infty}\left(J ; H^{1}\left(\Omega_{B, F}^{\varepsilon_{0} \cdots \varepsilon_{i-1}}\right)\right)}+\left\|\rho_{i-1, t}^{\left(\varepsilon_{0} \cdots \varepsilon_{i-1}\right)}\right\|_{L^{2}\left(\Omega_{B, F}^{\left.\varepsilon_{0} \cdots \varepsilon_{i-1} \times J\right)}\right.} \\
\leq C\left(\delta_{i, 1}\|S\|_{L^{2}(\Omega \times J)}+\left\|\rho_{\text {init }}\right\|_{H^{1}(\Omega)}\right), \\
\left\|\rho_{i}^{h\left(\varepsilon_{0} \cdots \varepsilon_{i-1}\right)}\right\|_{L^{2}\left(\Omega_{B, B}^{\varepsilon_{\left.\varepsilon^{\cdots} \cdots \varepsilon_{i-1} \times J\right)}}\right.}+\left\|\rho_{i, t}^{h\left(\varepsilon_{0} \cdots \varepsilon_{i-1}\right)}\right\|_{L^{2}\left(\Omega_{B, B}^{\left.\varepsilon_{0} \cdots \varepsilon_{i-1} \times J\right)}\right.} \leq C\left\|\rho_{\text {init }}\right\|_{H^{1}(\Omega)^{\prime}}, \\
\varepsilon_{i-1}\left\|\nabla \rho_{i}^{h\left(\varepsilon_{0} \cdots \varepsilon_{i-1}\right)}\right\|_{L^{\infty}\left(J ; L^{2}\left(\Omega^{\left.\left.\varepsilon_{0} \cdots \varepsilon_{i-1} \times J\right)\right)}\right.\right.} \leq C\left\|\rho_{\text {init }}\right\|_{H^{1}(\Omega)^{\prime}}
\end{gathered}
$$

and for $j=i+1, \ldots, N$,

$$
\begin{aligned}
& \left\|\rho_{j}^{h\left(\varepsilon_{0} \cdots \varepsilon_{i-1}\right)}\right\|_{L^{\infty}\left(J ; L^{2}\left(\Omega \times Y_{0 B} \times \cdots \times Y_{(j-2) B} ; H^{1}\left(Y_{(j-1) B} \times J\right)\right)\right)} \\
& \quad+\left\|\rho_{j, t}^{h\left(\varepsilon_{0} \cdots \varepsilon_{i-1}\right)}\right\|_{L^{2}\left(\Omega \times Y_{0 B} \times \cdots \times Y_{(j-1) B}\right)} \leq C\left\|\rho_{\text {init }}\right\|_{H^{1}(\Omega)} .
\end{aligned}
$$


Proof. This can be established by a straightforward application of [23, Propositions I.4.1, III.2.1, III.2.5], a standard fixed-point argument, and the usual energy and Dirichlet problem estimates for parabolic equations.

A similar kind of arguments also works to prove the following theorem.

Theorem 7.2. Problem (7.1), (7.3) is well posed in

$$
\begin{aligned}
L^{\infty}(J ; & \left.H^{1}\left(\Omega_{B, B}^{\varepsilon_{0} \cdots \varepsilon_{i-1}}\right)\right) \cap L^{2}\left(\Omega_{B, B}^{\varepsilon_{0} \cdots \varepsilon_{i-1}} ; H^{1}(J)\right) \\
\times & {\left[\prod_{j=i+1}^{N} L^{\infty}\left(J ; L^{2}\left(\Omega \times Y_{0 B} \times \cdots \times Y_{(j-2) B} ; H^{1}\left(Y_{(j-1) B} \times J\right)\right)\right)\right.} \\
& \left.\cap L^{2}\left(\Omega \times Y_{0 B} \times \cdots \times Y_{(j-1) B} ; H^{1}(J)\right)\right]
\end{aligned}
$$

In order to identify the intermediate source terms which do not appear in the triple-porosity model, the following lemma is essential.

LeMMA 7.3. Let $F^{\varepsilon_{0} \cdots \varepsilon_{i-1}}, \varphi \in L^{2}\left(\Omega \times Y_{0 B} \times \cdots \times Y_{(j-1) B} \times J\right)$. Assume that $F^{\varepsilon_{0} \cdots \varepsilon_{i-1}} \rightarrow F^{\varepsilon_{0} \cdots \varepsilon_{i-2}}$ weakly in $L^{2}\left(\Omega \times Y_{0 B} \times \cdots \times Y_{(j-1) B} \times J\right)$. Then, for $j=$ $i, \ldots, N-1$,

$$
\begin{gathered}
\int_{J \times \Omega \times Y_{0 B} \times \cdots \times Y_{(j-1) B}}\left[\int _ { Y _ { 0 } \times \cdots \times Y _ { i - 1 } } F ^ { \varepsilon _ { 0 } \cdots \varepsilon _ { i - 1 } } \left(\sum_{k=0}^{i-1}\left(\varepsilon_{k} y_{k+1}+c^{k, \varepsilon_{k}}\left(x_{k}\right)\right) \mathbf{e}_{k}\right.\right. \\
\left.\left.+\sum_{k=i}^{j} x_{k} \mathbf{e}_{k}+t \mathbf{e}_{j+1}\right) d y_{i} \cdots d y_{1}\right] \\
\times \varphi\left(x_{0}, \ldots, x_{j}, t\right) d x_{j} \cdots d x_{0} d t \stackrel{\varepsilon_{i-1} \rightarrow 0}{\longrightarrow}\left|Y_{i-1}\right| \\
\times \int_{J \times \Omega \times Y_{0 B} \times \cdots \times Y_{(j-1) B}}\left[\int_{Y_{0} \times \cdots \times Y_{i-2}} F^{\varepsilon_{0} \cdots \varepsilon_{i-2}}\right. \\
\times\left(\sum_{k=0}^{i-2}\left(\varepsilon_{k} y_{k+1}+c^{k, \varepsilon_{k}}\left(x_{k}\right)\right) \mathbf{e}_{k}\right. \\
\left.\left.+\sum_{k=i-1}^{j} x_{k} \mathbf{e}_{k}+t \mathbf{e}_{j+1}\right) d y_{i-1} \cdots d y_{1}\right] \\
\times \varphi\left(x_{0}, \ldots, x_{j}, t\right) d x_{j} \cdots d x_{0} d t .
\end{gathered}
$$


Proof. Using Fubini's theorem, we interchange the integration spaces in the following convenient way:

$$
\begin{aligned}
& \int_{J \times \Omega \times Y_{0 B} \times \cdots \times Y_{(j-1) B}} \int_{Y_{0} \times \cdots \times Y_{i-1}} F^{\varepsilon_{0} \cdots \varepsilon_{i-1}}\left(\sum_{k=0}^{i-1}\left(\varepsilon_{k} y_{k+1}+c^{k, \varepsilon_{k}}\left(x_{k}\right)\right) \mathbf{e}_{k}\right. \\
& \left.+\sum_{k=i}^{j} x_{k} \mathbf{e}_{k}+t \mathbf{e}_{j+1}\right) d y_{i} \cdots d y_{1} \\
& \times \varphi\left(x_{0}, \ldots, x_{j}, t\right) d x_{j} \cdots d x_{0} d t \\
& =\int_{J \times \Omega \times Y_{0 B} \times \cdots \times Y_{(i-3) B} \times Y_{(i-1) B} \times \cdots \times Y_{(j-1) B}} \\
& \times \int_{Y_{0} \times \cdots \times Y_{i-2}}\left[\int_{Y_{(i-2) B} \times Y_{(i-1)}} F^{\varepsilon_{0} \cdots \varepsilon_{i-1}}\left(\ldots, \varepsilon_{i-1} y_{i}+c^{(i-1), \varepsilon_{i-1}}\left(x_{i-1}\right), \ldots\right)\right. \\
& \left.\times \varphi\left(\ldots, x_{i-1}, \ldots\right) d y_{i} d x_{i-1}\right] \\
& \times d y_{i-1} \cdots d y_{1} d x_{j} \cdots d x_{i} d x_{i-2} \cdots d x_{0} d t \\
& =\int_{J \times \Omega \times Y_{0 B} \times \cdots \times Y_{(i-3) B} \times Y_{(i-1) B} \times \cdots \times Y_{(j-1) B}} \\
& \times \int_{Y_{0} \times Y_{0 B} \times \cdots \times Y_{i-2}}\left[\int_{Y_{(i-2) B} \times Y_{(i-1)}} F^{\varepsilon_{0} \cdots \varepsilon_{i-1}}\left(\ldots, x_{i-1}, \ldots\right)\right. \\
& \times \varphi\left(\ldots, \varepsilon_{i-1} y_{i}+c^{(i-1), \varepsilon_{i-1}}\left(x_{i-1}\right),\right. \\
& \text {...) } \left.d y_{i} d x_{i-1}\right] \\
& \times d y_{i-1} \cdots d y_{1} d x_{j} \cdots d x_{i} d x_{i-2} \cdots d x_{0} d t \\
& \stackrel{\varepsilon_{i-1} \rightarrow 0}{\longrightarrow} \int_{J \times \Omega \times Y_{0 B} \times \cdots \times Y_{(i-3) B} \times Y_{(i-1) B} \times \cdots \times Y_{(j-1) B}} \\
& \times \int_{Y_{0} \times \cdots \times Y_{i-2}}\left[\int_{Y_{(i-2) B} \times Y_{(i-1)}} F^{\varepsilon_{0} \cdots \varepsilon_{i-2}}\left(\ldots, x_{i-1}, \ldots\right)\right. \\
& \left.\times \varphi\left(\ldots, x_{i-1}, \ldots\right) d y_{i} d x_{i-1}\right] \\
& \times d y_{i-1} \cdots d y_{1} d x_{j} \cdots d x_{i} d x_{i-2} \cdots d x_{0} d t \\
& =\left|Y_{i-1}\right| \int_{J \times \Omega \times Y_{0 B} \times \cdots \times Y_{(i-3) B} \times Y_{(i-1) B} \times \cdots \times Y_{(j-1) B}} \\
& \times \int_{Y_{0} \times \cdots \times Y_{i-2}}\left[\int_{Y_{(i-2) B}} F^{\varepsilon_{0} \cdots \varepsilon_{i-2}}\left(\ldots, x_{i-1}, \ldots\right)\right. \\
& \left.\times \varphi\left(\ldots, x_{i-1}, \ldots\right) d x_{i-1}\right] \\
& \times d y_{i-1} \cdots d y_{1} d x_{j} \cdots d x_{i} d x_{i-2} \cdots d x_{0} d t
\end{aligned}
$$




$$
\begin{aligned}
&=\left|Y_{i-1}\right| \\
& \times \int_{J \times \Omega \times \cdots \times \cdots \times Y_{(j-1) B}}\left[\int _ { Y _ { 0 } \times \cdots \times Y _ { i - 2 } } F ^ { \varepsilon _ { 0 } \cdots \varepsilon _ { i - 2 } } \left(\sum_{k=0}^{i-2}\left(\varepsilon_{k} y_{k+1}+c^{k, \varepsilon_{k}}\left(x_{k}\right)\right) \mathbf{e}_{k}\right.\right. \\
&\left.\left.+\sum_{k=i-1}^{j} x_{k} \mathbf{e}_{k}+t \mathbf{e}_{j+1}\right) d y_{i-1} \cdots d y_{1}\right] \\
& \times \varphi\left(x_{0}, \ldots, x_{j}, t\right) d x_{j} \cdots d x_{0} d t
\end{aligned}
$$

where Lemma 2.5 gives the second equality, and the weak convergence of $F^{\varepsilon_{0} \cdots \varepsilon_{i-1}}$ to $F^{\varepsilon_{0} \cdots \varepsilon_{i-2}}$ and the strong convergence of $\phi\left(x_{0}, \ldots, \varepsilon_{i-1} y_{i}+\right.$ $\left.c^{(i-1), \varepsilon_{i-1}}\left(x_{i-1}\right), \ldots, x_{j}, t\right)$ to $\phi\left(x_{0}, \ldots, x_{i-1}, \ldots, x_{j}, t\right)$ as $\varepsilon_{i-1} \rightarrow 0$ give the convergence result. The volume $\left|Y_{i-1}\right|$ appears as a result of the integrand's independence on the variable $y_{i-2}$ after $\varepsilon_{i-1} \rightarrow 0$. Fubini's theorem gives the final equality.

Corollary 7.4. With the definition of $S_{j}^{h\left(\varepsilon_{0} \cdots \varepsilon_{i-1}\right)}$ in (7.4),

$$
S_{j}^{h\left(\varepsilon_{0} \cdots \varepsilon_{i-1}\right)} \rightarrow S_{j}^{h\left(\varepsilon_{0} \cdots \varepsilon_{i-2}\right)} \text { weakly as } \varepsilon_{i-1} \longrightarrow 0
$$

Notice now that

$$
\begin{aligned}
& \left(L_{i}^{h\left(\varepsilon_{0} \cdots \varepsilon_{i-1}\right)}\right)^{\sim(i)} \\
& =\sum_{\alpha=i}^{N-1} \frac{1}{\left|Y_{0}\right| \cdots\left|Y_{\alpha}\right|} \\
& \quad \times \int_{Y_{0} \times \cdots \times Y_{i-1} \times Y_{i B} \times \cdots \times Y_{\alpha B}} \phi_{\alpha+1}^{h}\left(y_{\alpha+1}\right) \frac{\partial \rho_{\alpha+1}^{h\left(\varepsilon_{0} \cdots \varepsilon_{i-1}\right)}}{\partial t} \\
& \times\left(\sum_{k=0}^{i-1}\left(\varepsilon_{k} y_{k+1}+c^{k, \varepsilon_{k}}\left(x_{k}\right)\right) \mathbf{e}_{k}+x_{i} \mathbf{e}_{i}\right. \\
& \left.\quad+\sum_{k=i+1}^{\alpha+1} y_{k} \mathbf{e}_{k}+t \mathbf{e}_{\alpha+2}\right) d y_{\alpha+1} \cdots d y_{1} \\
& \equiv S_{i}^{h\left(\varepsilon_{0} \cdots \varepsilon_{i-1}\right)} .
\end{aligned}
$$

We observe that this definition of $S_{i}^{h\left(\varepsilon_{0} \cdots \varepsilon_{i-1}\right)}$ agrees with the one given by (7.4), with the empty product in the numerator of the coefficient there 
replaced by 1 . Thus, by Lemma 7.3, with $j=i$, we have the following weak convergence result in $L^{2}(D)$ :

$$
\begin{gathered}
\left(L_{i}^{h\left(\varepsilon_{0} \cdots \varepsilon_{i-1}\right)}\right)^{\sim(i)} \stackrel{\varepsilon_{i-1} \rightarrow 0}{\longrightarrow} \sum_{\alpha=i}^{N-1} \frac{\left|Y_{i-1}\right|}{\left|Y_{0}\right| \cdots\left|Y_{\alpha}\right|} \\
\times \int_{Y_{0} \times \cdots \times Y_{i-2} \times Y_{i B} \times \cdots \times Y_{\alpha B}} \phi_{\alpha+1}^{h}\left(y_{\alpha+1}\right) \frac{\partial \rho_{\alpha+1}^{h\left(\varepsilon_{0} \cdots \varepsilon_{i-1}\right)}}{\partial t} \\
\times\left(\sum_{k=0}^{i-2}\left(\varepsilon_{k} y_{k+1}+c^{k, \varepsilon_{k}}\left(x_{k}\right)\right) \mathbf{e}_{k}+\sum_{k=i-1}^{i} x_{k} \mathbf{e}_{k}\right. \\
\left.\quad+\sum_{k=i+1}^{\alpha+1} y_{k} \mathbf{e}_{k}+t \mathbf{e}_{\alpha+2}\right) d y_{\alpha+1} \cdots d y_{1} \\
\equiv S_{i}^{h\left(\varepsilon_{0} \cdots \varepsilon_{i-2}\right) .}
\end{gathered}
$$

The permeability tensors that arise in the intermediate stages of homogenization are given by

$$
\left(\mathbf{K}_{i-1}^{h\left(\varepsilon_{0} \cdots \varepsilon_{i-2}\right)}\right)_{\alpha \beta}=K_{i-1}^{\varepsilon_{0} \cdots \varepsilon_{i-2}}\left(\omega_{\alpha \beta}^{i-1}+\frac{\left|Y_{(i-1) F}\right|}{\left|Y_{i-1}\right|} \delta_{\alpha \beta}\right) .
$$

In the above definition, the geometry of the fractures enters the equations via $\boldsymbol{\omega}^{i-1} \equiv\left(\omega^{i-1}\right)_{\alpha \beta}$, which is defined by the $Y_{i-1}$-periodic solution $\omega_{\beta}^{i-1}$, modulo constants, of the following Neumann problem:

$$
\begin{gathered}
\Delta_{x_{i}} w_{\beta}^{i-1}=0 \quad \text { in } Y_{(i-1) F}, \quad \nabla_{x_{i}} \omega_{\beta}^{i-1} \cdot v=-\mathbf{e}_{\beta} \cdot v=-v_{\beta} \quad \text { on } \partial Y_{(i-1) B}, \\
\omega_{\alpha \beta}^{i-1}=\frac{1}{\left|Y_{i-1}\right|} \int_{Y_{(i-1) F}} \partial_{i, \alpha} \omega_{\beta}^{i-1}\left(x_{i}\right) d x_{i}
\end{gathered}
$$

where $\partial_{i, \alpha}=\partial / \partial x_{i, \alpha}$.

Finally, define

$$
\phi_{i-1}^{h\left(\varepsilon_{0} \cdots \varepsilon_{i-2}\right)} \equiv \frac{\left|Y_{(i-1) F}\right|}{\left|Y_{i-1}\right|} \phi_{i-1}^{\varepsilon_{0} \cdots \varepsilon_{i-2}}, \quad \rho_{i-1}^{h\left(\varepsilon_{0} \cdots \varepsilon_{i-2}\right)} \equiv \frac{\left|Y_{i-1}\right|}{\left|Y_{(i-1) F}\right|} \hat{\rho}_{i-1}^{\varepsilon_{0} \cdots \varepsilon_{i-2}}
$$

By adapting the arguments employed in the homogenization of the triple-porosity model, using in addition Corollary 7.4 and (7.15), we arrive at the following theorem.

Theorem 7.5. For $i=1, \ldots, N$, let $\left(\rho_{i-1}^{\left(\varepsilon_{0} \cdots \varepsilon_{i-1}\right)}, \rho_{i}^{h\left(\varepsilon_{0} \cdots \varepsilon_{i-1}\right)}, \ldots, \rho_{N}^{h\left(\varepsilon_{0} \cdots \varepsilon_{i-1}\right)}\right)$ be the unique weak solution to problem (7.1) (with the new boundary condition (7.5)), 
(7.3), and (7.6). Then, as $\varepsilon_{i-1} \rightarrow 0$, the following limits hold weakly in the indicated spaces:

$$
\begin{aligned}
& \chi_{\Omega_{B, F}^{\varepsilon_{0} \cdots \varepsilon_{i-1}}} \rho_{i-1}^{\left(\varepsilon_{0} \cdots \varepsilon_{i-1}\right)} \rightarrow \widehat{\rho}_{i-1}^{\varepsilon_{0} \cdots \varepsilon_{i-2}} \text { in } H^{1}\left(J ; L^{2}\left(\Omega_{B, B}^{\varepsilon_{0} \cdots \varepsilon_{i-2}}\right)\right), \\
& \chi_{\Omega_{B, F}^{\varepsilon_{0} \cdots \varepsilon_{i-1}}} \frac{K_{i-1}^{\varepsilon_{0} \cdots \varepsilon_{i-2}}}{\mu C} \nabla_{x_{0}} \rho_{i-1}^{\left(\varepsilon_{0} \cdots \varepsilon_{i-1}\right)} \rightarrow \frac{\left|Y_{i-1}\right|}{\left|Y_{(i-1) F}\right|} \mathbf{K}_{i-1}^{h\left(\varepsilon_{0} \cdots \varepsilon_{i-2}\right)} \nabla_{x_{0}} \hat{\rho}_{i-1}^{\varepsilon_{0} \cdots \varepsilon_{i-2}} \\
& \text { in } L^{2}\left(\Omega_{B, B}^{\varepsilon_{0} \cdots \varepsilon_{i-2}} \times J\right) \text {, } \\
& \left(\rho_{i}^{h\left(\varepsilon_{0} \cdots \varepsilon_{i-1}\right)}\right)^{\sim(i)} \rightarrow \rho_{i}^{h\left(\varepsilon_{0} \cdots \varepsilon_{i-2}\right)} \text { in } L^{2}\left(\Omega \times Y_{0 B} \times \cdots \times Y_{(i-2) B} ; H^{1}\left(Y_{(i-1) B} \times J\right)\right) \text {, } \\
& \text { and for } j=i+1, \ldots, N \text {, } \\
& \rho_{j}^{h\left(\varepsilon_{0} \cdots \varepsilon_{i-1}\right)} \rightarrow \rho_{j}^{h\left(\varepsilon_{0} \cdots \varepsilon_{i-2}\right)} \quad \text { in } L^{2}\left(\Omega \times Y_{0 B} \times \cdots \times Y_{(j-2) B} ; H^{1}\left(Y_{(j-1) B} \times J\right)\right)
\end{aligned}
$$

Moreover, if

$$
\rho_{i-1}^{h\left(\varepsilon_{0} \cdots \varepsilon_{i-2}\right)}=\frac{\left|Y_{i-1}\right|}{\left|Y_{(i-1) F}\right|} \widehat{\rho}_{i-1}^{\varepsilon_{0} \cdots \varepsilon_{i-2}},
$$

then $\left(\rho_{i-1}^{h\left(\varepsilon_{0} \cdots \varepsilon_{i-2}\right)}, \ldots, \rho_{N}^{h\left(\varepsilon_{0} \cdots \varepsilon_{i-2}\right)}\right)$ is the unique weak solution of problem (7.1), (7.3) with $i$ replaced by $i-1$. In particular, as $\varepsilon_{0} \rightarrow 0$, the following weak limits hold:

$$
\begin{aligned}
& \chi_{\Omega_{F}^{\varepsilon_{0}}} \rho_{0}^{\varepsilon_{0}} \rightarrow \widehat{\rho}_{0} \quad \text { in } H^{1}\left(J ; L^{2}(\Omega)\right), \\
& \chi \Omega_{B}^{\varepsilon_{0}} \frac{K_{0}^{\varepsilon_{0}}}{\mu c} \nabla \rho_{0}^{\varepsilon_{0}} \rightarrow \frac{\left|Y_{0}\right|}{\left|Y_{0 F}\right|} K_{0}^{h} \nabla \hat{\rho}_{0} \quad \text { in } L^{2}(\Omega \times J), \\
& \left(\rho_{1}^{h\left(\varepsilon_{0}\right)}\right)^{\sim(1)} \rightarrow \rho_{1} \quad \text { in } L^{2}\left(\Omega ; H^{1}\left(Y_{0 B} \times J\right)\right),
\end{aligned}
$$

and for $j=2, \ldots, N$,

$$
\rho_{j}^{h\left(\varepsilon_{0}\right)} \rightarrow \rho_{j} \quad \text { in } L^{2}\left(\Omega \times Y_{0 B} \times \cdots \times Y_{(j-2) B} ; H^{1}\left(Y_{(j-1) B} \times J\right)\right)
$$

Moreover, if

$$
\rho_{0}=\frac{\left|Y_{0}\right|}{\left|Y_{0 F}\right|} \widehat{\rho}_{0}, \quad S^{h}=\frac{\left|Y_{0 F}\right|}{\left|Y_{0}\right|} S
$$


then $\left(\rho_{0}, \ldots, \rho_{N}\right)$ is the unique weak solution of the following system of final homogenized equations:

$$
\begin{gathered}
\phi_{0}^{h} \frac{\partial \rho_{0}}{\partial t}-\nabla_{x_{0}} \cdot\left(\frac{\mathbf{K}_{0}^{h}}{\mu c} \nabla_{x_{0}} \rho_{0}\right) \\
+\sum_{k=1}^{N} \frac{1}{\left|Y_{0}\right| \cdots\left|Y_{k-1}\right|} \int_{Y_{0 B} \times \cdots \times Y_{(k-1) B}} \phi_{k}^{h} \frac{\partial \rho_{k}}{\partial t} d x_{k} \cdots d x_{1}=S^{h} \quad \text { in } \Omega \times J, \\
\frac{\mathbf{K}_{0}^{h}}{\mu c} \nabla_{x_{0}} \rho_{0} \cdot n_{\Omega}=0 \quad \text { on } \partial \Omega \times J, \\
\rho_{0}=\rho_{\text {init }} \quad \text { in } \Omega \times\{0\},
\end{gathered}
$$

and for every $x_{j-1} \in Y_{(j-2) B}$, there exists a block $Y_{(j-1) B}$ such that $\rho_{j}$ solves the following initial-boundary value problem for $j=1, \ldots, N$ :

$$
\begin{aligned}
& \phi_{j}^{h} \frac{\partial \rho_{j}}{\partial t}-\nabla_{x_{j}} \cdot\left(\frac{\mathbf{K}_{j}^{h}}{\mu c} \nabla_{x_{j}} \rho_{j}\right) \\
& \quad+\sum_{k=j}^{N-1} \frac{1}{\left|Y_{j}\right| \cdots\left|Y_{k}\right|} \int_{Y_{j B} \times \cdots \times Y_{k B}} \phi_{k+1}^{h} \frac{\partial \rho_{k+1}}{\partial t} d x_{k+1} \cdots d x_{j+1} \\
& =0 \quad \text { in } \Omega \times Y_{0 B} \times \cdots \times Y_{(j-1) B} \times J, \\
& \rho_{j}=\rho_{j-1} \quad \text { on } \Omega \times Y_{0 B} \times \cdots \times Y_{(j-2) B} \times \partial Y_{(j-1) B} \times J, \\
& \rho_{j}=\rho_{\text {init }} \quad \text { in } \Omega \times Y_{0 B} \times \cdots \times Y_{(j-1) B} \times\{0\} .
\end{aligned}
$$

\section{Concluding remarks}

Each equation in the final homogenized system of the $(N+1)$-scale problem contains interchange terms from the relatively smaller scales. This shows that the recursive homogenization procedure captures the microscale effects. Instead, if the entire reservoir was homogenized in a straightforward manner as was done in $[1,10]$, a single-porosity model with an average permeability would result. That would be inadequate here since the porous structures are quite distinct. Here, we retain the fine microscopic structures, yet we average their effects.

It is worth noting that instead of putting the external source term solely on the first level of fractures, we could have easily defined it on each level. If this is desired, then the above analysis still holds with the modification that in the final homogenized system of equations, the external source term would appear on each level with no modification from the homogenization. 


\section{Acknowledgment}

This research is funded by an Oakland University Research Grant.

\section{References}

[1] B. Amaziane and A. Bourgeat, Effective behavior of two-phase flow in heterogeneous reservoir, Numerical Simulation in Oil Recovery (Minneapolis, Minn., 1986) (M. F. Wheeler, ed.), IMA Vol. Math. Appl., vol. 11, Springer, New York, 1988, pp. 1-22.

[2] T. Arbogast, The double porosity model for single phase flow in naturally fractured reservoirs, Numerical Simulation in Oil Recovery (Minneapolis, Minn., 1986) (M. F. Wheeler, ed.), IMA Vol. Math. Appl., vol. 11, Springer, New York, 1988, pp. 23-45.

[3] - Analysis of the simulation of single phase flow through a naturally fractured reservoir, SIAM J. Numer. Anal. 26 (1989), no. 1, 12-29.

[4] - On the simulation of incompressible, miscible displacement in a naturally fractured petroleum reservoir, RAIRO Modél. Math. Anal. Numér. 23 (1989), no. $1,5-51$.

[5] T. Arbogast, J. Douglas Jr., and U. Hornung, Derivation of the double porosity model of single phase flow via homogenization theory, SIAM J. Math. Anal. 21 (1990), no. 4, 823-836.

[6] T. Arbogast, J. Douglas Jr., and J. E. Santos, Two-phase immiscible flow in naturally fractured reservoirs, Numerical Simulation in Oil Recovery (Minneapolis, Minn., 1986) (M. F. Wheeler, ed.), IMA Vol. Math. Appl., vol. 11, Springer, New York, 1988, pp. 47-66.

[7] G. I. Barenblatt, V. M. Entov, and V. M. Ryzhik, Theory of fluid flows through natural rocks, Theory and Applications of Transport in Porous Media (J. Bear, ed.), Kluwer Academic Publishers, Dordrecht, 1990.

[8] G. I. Barenblatt, I. P. Zheltov, and I. N. Kochina, Basic concepts in the theory of seepage of homogeneous liquids in fissured rocks [strata], Prikl. Mat. Mekh. 24 (1960), 852-864, translated in J. Appl. Math. Mech. 24 (1960), 1286-1303.

[9] A. Bensoussan, J.-L. Lions, and G. Papanicolaou, Asymptotic Analysis for Periodic Structures, Studies in Mathematics and Its Applications, vol. 5, NorthHolland Publishing, Amsterdam, 1978.

[10] A. Bourgeat, Homogenization of two-phase flow equations, Nonlinear Functional Analysis and Its Applications, Part 1 (Berkeley, Calif, 1983), Proc. Sympos. Pure Math., vol. 45, American Mathematical Society, Rhode Island, 1986, pp. 157-163.

[11] A. Bourgeat, A. Mikelić, and S. Wright, Stochastic two-scale convergence in the mean and applications, J. reine angew. Math. 456 (1994), 19-51.

[12] A.-P. Calderón, Lebesgue spaces of differentiable functions and distributions, Proc. Sympos. Pure Math., Vol. IV, American Mathematical Society, Rhode Island, 1961, pp. 33-49.

[13] J. Douglas Jr. and T. Arbogast, Dual-porosity models for flow in naturally fractured reservoirs, Dynamics of Fluids in Hierarchical Porous Formations, Academic Press, London, 1990, pp. 177-221. 


\section{Analysis of a multiple-porosity model}

[14] J. Douglas Jr., T. Arbogast, P. J. Paes Leme, J. L. Hensley, and N. P. Nunes, Immiscible displacement in vertically fractured reservoirs, Transport in Porous Media 12 (1993), 73-106.

[15] J. Douglas Jr., C.-S. Huang, and A. M. Spagnuolo, The approximation of nuclear contaminant transport in porous media, J. Comput. Appl. Math. 21 (2002), no. $2,409-428$.

[16] J. Douglas Jr., M. Kischinhevsky, P. J. Paes Leme, and A. M. Spagnuolo, A multiple-porosity model for a single-phase flow through naturally-fractured porous media, J. Comput. Appl. Math. 17 (1998), no. 1, 19-48.

[17] J. Douglas Jr., P. J. Paes Leme, T. Arbogast, and T. Schmitt, Simulation of flow in naturally fractured petroleum reservoirs, Proceedings of the Ninth SPE Symposium on Reservoir Simulation, SPE, vol. 16019, Society of Petroleum Engineers, Texas, 1987, pp. 271-279.

[18] J. Douglas Jr., P. J. Paes Leme, and J. L. Hensley, A limit form of the equations for immiscible displacement in a fractured reservoir, Transport in Porous Media 6 (1991), 549-565.

[19] J. Douglas Jr., M. Peszyńska, and R. E. Showalter, Single phase flow in partially fissured media, Transport in Porous Media 28 (1997), 285-306.

[20] J. Douglas Jr. and A. M. Spagnuolo, The transport of nuclear contamination in fractured porous media, J. Korean Math. Soc. 38 (2001), no. 4, 723-761.

[21] A. Fasano, A. Mikelić, and M. Primicerio, Homogenization of flows through porous media with permeable grains, Adv. Math. Sci. Appl. 8 (1998), no. 1, $1-31$.

[22] E. Sánchez-Palencia, Nonhomogeneous Media and Vibration Theory, Lecture Notes in Physics, vol. 127, Springer-Verlag, Berlin, 1980.

[23] R. E. Showalter, Monotone Operators in Banach Space and Nonlinear Partial Differential Equations, Mathematical Surveys and Monographs, vol. 49, American Mathematical Society, Rhode Island, 1997.

[24] A. M. Spagnuolo, Approximation of nuclear contaminant transport through porous media, Ph.D. thesis, Center for Applied Mathematics, Purdue University, Indiana, 1998.

[25] J. E. Warren and P. J. Root, The behavior of naturally fractured reservoirs, Soc. Pet. Eng. J. 3 (1963), 245-255.

Anna Maria Spagnuolo: Department of Mathematics and Statistics, Oakland University, Rochester, MI 48309-4485, USA

E-mail address: spagnuol@oakland.edu

Steve Wright: Department of Mathematics and Statistics, Oakland University, Rochester, MI 48309-4485, USA

E-mail address: wright@oakland.edu 


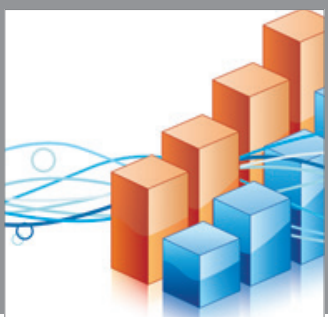

Advances in

Operations Research

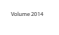

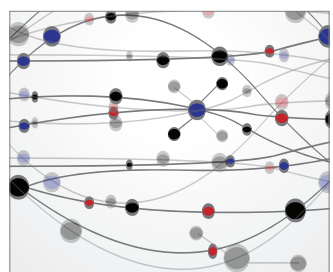

\section{The Scientific} World Journal
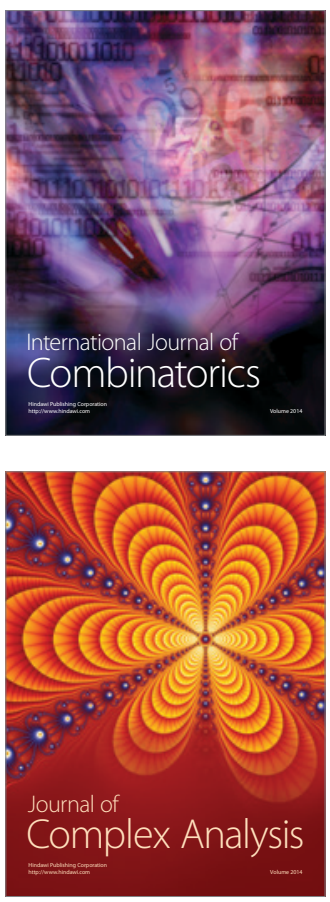

International Journal of

Mathematics and

Mathematical

Sciences
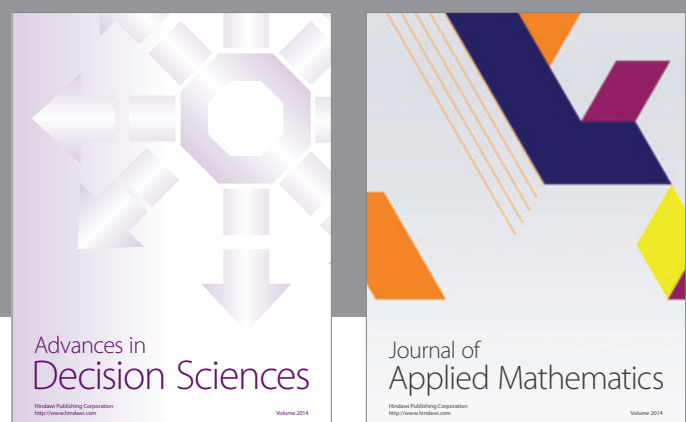

Journal of

Applied Mathematics
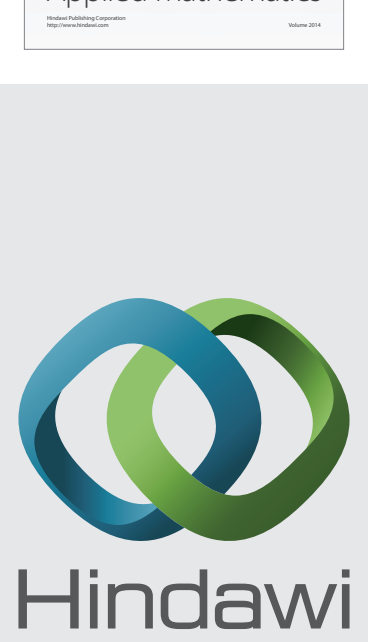

Submit your manuscripts at http://www.hindawi.com
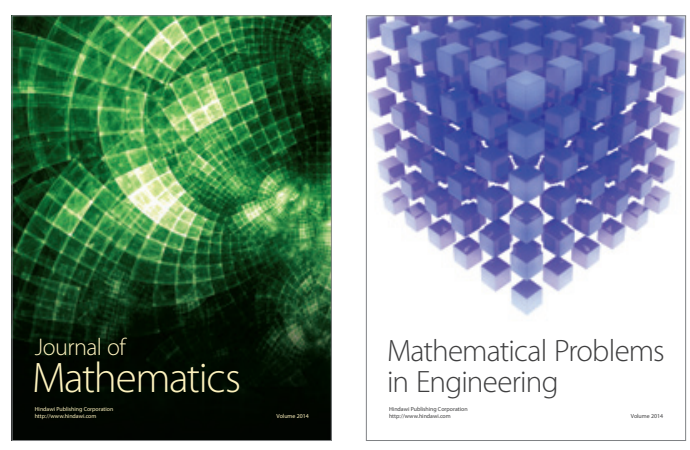

Mathematical Problems in Engineering
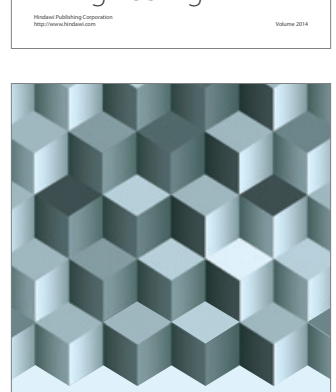

Journal of

Function Spaces
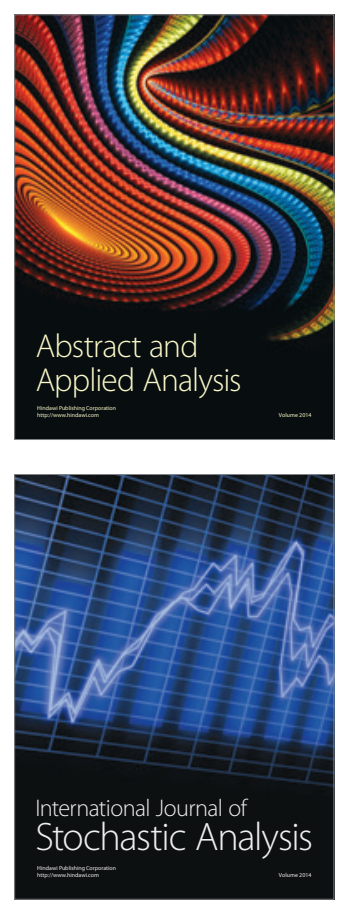

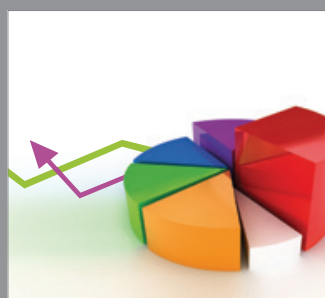

ournal of

Probability and Statistics

Promensencen
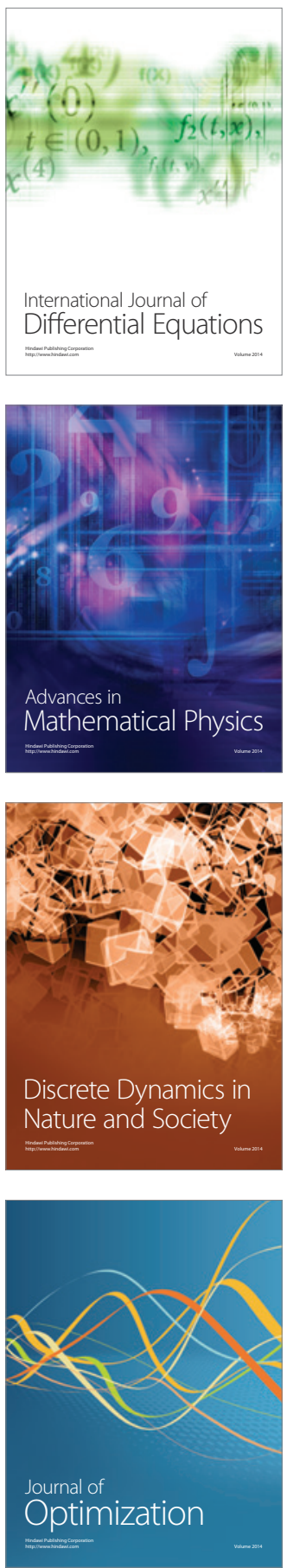